\title{
Derivation via free energy conservation constraints of gyrofluid equations with finite-gyroradius electromagnetic nonlinearities
}

\author{
B. Scott* \\ Max-Planck-Institut für Plasmaphysik, \\ Euratom Association, D-85748 Garching, Germany
}

(Dated: Mar 2010)

\begin{abstract}
The derivation of electromagnetic gyrofluid equations is made systematic by using the Hermite polynomial form of the underlying delta-f gyrokinetic distribution function. The gyrokinetic freeenergy functional is explicitly used to set up the model. The gyrofluid free energy follows directly. The interaction term in the gyrokinetic Lagrangian is used to obtain the gyrofluid counterpart, from which the polarisation equation follows. One closure rule is decided for taking moments over the kinetic gyroaveraging operator. These steps fix the rest of the derivation of the conservative part of the gyrofluid equations. Dissipation is then added in a form to obtain positive definite dissipation and to obtain the collisional fluid equations in their appropriate limit. Existing results are recovered, with the addition of a completely consistent model for finite gyroradius effects in the nonlinearities responsible for magnetic reconnection.
\end{abstract}

PACS numbers: 52.65.Tt, 52.35.Ra, 52.30.-q, 52.25.Fi

*email: bds@ipp.mpg.de;

URL: http://www.rzg.mpg.de/ bds/ 


\section{INTRODUCTION}

Gyrofluid equations in toroidal geometry were originally derived for small-amplitude disturbances to treat linear instabilities [1]. Nonlinear terms were taken from an earlier version derived in slab geometry [2]. Landau damping dissipation, resulting from phase mixing into arbitrarily small velocity space structures, was taken from a previous closure dissipation model [3]. Electromagnetic versions were also derived [4, 5]. The original idea behind gyrofluid equations, derived in two-dimensional slab geometry, was for the density of a fluid of gyrocenters rather than particles, with a polarisation equation tying the gyrocenter densities together in a statement of overall charge neutrality [6]. Polarisation results from the part of the space density arising from the gyrophase angle dependent part of the distribution function, while the gyrocenter density reflects the part independent of the gyrophase angle. This was already a result of gyrokinetic theory [7], which was originally formulated as a finite Larmor radius (FLR) correction to the drift kinetic equation [8]. The gyrofluid model rests on the gyrokinetic model, much in the same fashion that the more familiar fluid model rests on the Vlasov (or Boltzmann) equation for particles [9, 10].

The gyrokinetic theory itself, however, underwent a serious advance resulting from the use of Lie transformation techniques applied to the drift kinetic Lagrangian [11 13]. The Lagrangian was to be transformed to gyrocenter coordinates and only then the gyrokinetic equation was to be found in terms of the resulting Euler-Lagrange equations describing the motion of individual gyrocenters. The gyrofluid model, on the other hand, continued to be based upon the older gyrokinetic formulation, in which it was not always transparent what one had to keep in order to maintain consistency at a given ordering. Indeed, the original toroidal model had to be modified before it had a clearly consistent energy conservation theorem [14, 15]. The gyrokinetic theory was re-cast as a Lagrangian field theory [16, 17], and nonlinear nonlocal gyrofluid equations have also been formed in a similar manner [18, 19].

The delta-f form of the gyrokinetic theory also has its energy theorem [20], whose conserved quantity as a functional quadratic in all the dependent variables is better thought of as a free energy, similar to that of fluid equations [21 24]. This has been shown to be related to entropy [25]. Free energy for fluid equations has also been shown to be related to this entropy [26].

The original gyrofluid derivation for toroidal geometry led to some inconsistencies which 
caused fluctuation free energy not to be conserved. These were repaired by a construction method which can be thought of as somewhat arbitrary and/or artificial [15]. The free energy was determined by analogy to the fluid equations, leaving it open to the argument that it might not be fundamental. The key insight in the meantime was the connection made between the models, showing the gyrofluid moment variables can be cast in terms of an Hermite polynomial representation of the gyrokinetic distribution function. The polynomials are functions of the velocity space coordinates, and the coefficients of these are the gyrofluid moment variables. Given the gyrokinetic delta-f free energy functional, the gyrofluid one can be derived using this Hermite expansion. This was shown in Ref. [26] in terms of a simplified gyrofluid model without FLR corrections.

Herein, we systematise this procedure for the six-moment gyrofluid model of Refs. [1, 2, 4] which was corrected for energy conservation in Ref. [15]. But before that we need to show the relation to the only really first-principle model in the hierarchy: the total-f gyrokinetic equation obtained by Lie transforms [12, 13]. We start with that one, simplifying it mildly to obtain a computationally tractable form. Then we make the delta-f approximations in such a way as to keep energetic consistency intact. The total-f energy obtainable from the Noether theorem [16, 17] is replaced by the delta-f free energy functional referred to above. This delta-f equation and its free energy theorem then launch the derivation of the gyrofluid equations in local form (fully nonlinear equations, but constant background parameters, dependent on drift ordering). At the kinetic level, the gyrocenter part of the charge density in the polarisation equation is the same in the total-f and delta-f versions, and arises from the interaction term in the total-f Lagrangian. The FLR closure for the gyrofluid model is applied only once, and it is here. Ultimately, the role of polarisation is the same in all three models, and taking that into account allows energetic consistency to be maintained. Specifically, the same rules must be applied both to polarisation and to the moment variable equations [15]. The polarisation part of the charge density is the same in all models which use linearised polarisation, including the delta-f and gyrofluid levels. This obtains the polarisation equation, which at all levels can be used to re-cast the ExB kinetic energy in terms of gyrocenter charge potential energy. The part of the gyrofluid free energy due strictly to the moment variables follows from insertion of the Hermite polynomial representation into the delta-f form. The rest of the derivation is a mere matter of consistent application of the moments to the delta-f gyrokinetic equations. This part of the method is 
similar to that of Refs. [1, 2], but the results of Ref. [15] concerning the conservative part of the equations and the free energy are recovered without further pitfall. Dissipation is inserted manually as before.

Previous versions of gyrofluid equations have applied the FLR closure only to the electrostatic potential, leaving the electromagnetic response along. This is usually justified since the turbulence spectrum only extends down in scale as far as the ion gyroradius. Hence, FLR electron effects are small by the mass ratio. Moreover, ion dynamical contributions to the Ohm's Law are also finite mass ratio corrections. However, fluid-type equations such as these are also useful in the study of collisionless reconnection, which includes sufficiently small-scale phenomena that electron FLR effects may enter [27-30]. Herein, the FLR treatment is extended to the magnetic potential at the same level of sophistication as to the electrostatic potential. The parallel velocity and perp/parallel heat flux moments are mixed in the magnetic flutter disturbance and induction physics in the same way as the density and perpendicular temperature moments in the ExB advection and polarisation physics. This extension of the gyrofluid model represents the new result of this work.

\section{THE TOTAL-F AND DELTA-F FORMS OF THE GYROKINETIC MODEL}

The total-f Vlasov equation is a Hamiltonian bracket equation for the total distribution function $f$, a dependent variable over phase space coordinates $\{\mathbf{x}, z, w\}$ with the space part $\mathbf{x}$ given by field aligned coordinates $\{x, y, s\}$. Field aligning refers to a single nonvanishing contravariant component of the background magnetic field, in this case $B^{s}$, which identifies $s$ as the parallel coordinate. The velocity space part is given by the parallel velocity $z$ and magnetic moment $w$, with $f$ independent of the gyrophase angle $\theta$. As a starting point we use the result of the Lie transform theory version of the gyrokinetic expansion, specifically a version of the forms given in Eqs. (16,17,22-24) of Ref. [13], mildly simplified for computational use. The particle Lagrangian is given by

$$
L_{p}=\frac{e}{c} \mathbf{A}^{*} \cdot \frac{d \mathbf{x}}{d t}+w \frac{m c}{e} \frac{d \theta}{d t}-H
$$

where

$$
\mathbf{A}^{*}=\mathbf{A}+m z \frac{c}{e} \mathbf{b}
$$


where $\mathbf{A}$ and $\mathbf{b}$ and $B$ are the potential, unit vector, and field strength of the background magnetic field. The Hamiltonian $H$ is given by

$$
H=m \frac{z^{2}}{2}+w B+e J_{0} \phi-\frac{e^{2}}{2 B} \frac{\partial}{\partial w}\left[J_{0}\left(\phi^{2}\right)-\left(J_{0} \phi\right)^{2}\right]
$$

where $\phi$ is the electrostatic potential, $J_{0}$ is the gyroaveraging operator, and the contribution which is quadratic in $\phi$ is referred to as the gyroscreening potential. Formally, $J_{0}$ has the form in wavenumber space of multiplication of Fourier coefficients by the zeroth Bessel function $J_{0}\left(k_{\perp} \rho_{L}\right)$, where $\rho_{L}$ is the particle gyroradius given by $\rho_{L}=v_{\perp} / \Omega$, with gyrofrequency $\Omega=|e B / m c|$, or in terms of the coordinates by $\rho_{L}^{2}=2 w B /\left(m \Omega^{2}\right)$. The particle equations of motion are found from the Euler-Lagrange equations resulting from $L_{p}$,

$$
B_{\|}^{*} \frac{d \mathbf{x}}{d t}=z \mathbf{B}^{*}-\frac{c}{e B} \mathbf{F} \cdot \nabla H \quad m B_{\|}^{*} \frac{d z}{d t}=-\mathbf{B}^{*} \cdot \nabla H
$$

Drift tensor notation is used, with definitions

$$
\begin{gathered}
\mathbf{B}^{*}=\nabla \times \mathbf{A}^{*} \quad B_{\|}^{*}=\mathbf{b} \cdot \mathbf{B}^{*} \\
\mathbf{F}=\nabla \mathbf{A}-(\nabla \mathbf{A})^{T}
\end{gathered}
$$

It follows that

$$
\mathbf{F}=\epsilon \cdot \mathbf{B} \quad \nabla \times \mathbf{b}=-\nabla \cdot \frac{\mathbf{F}}{B} \quad \mathbf{B}^{*}=\mathbf{B}-m z \nabla \cdot \frac{c}{e} \frac{\mathbf{F}}{B}
$$

where $\epsilon$ is the rank-three Levi-Civita pseudotensor.

The polarisation equation is found by extending the Lagrangian to obtain one for the entire particles/field system using the methods of Refs. [16, 17],

$$
L=\sum_{\mathrm{sp}} \int d \Lambda f\left[\frac{e}{c} \mathbf{A}^{*} \cdot \frac{d \mathbf{x}}{d t}+w \frac{m c}{e} \frac{d \theta}{d t}-H\right]
$$

where the sum is over particle species, $f$ is the distribution function for particles of each species, $L_{p}$ multiplies $f$ expressed in phase space coordinates, $H$ is given in Eq. (3), and the phase space, velocity space, and configuration space integration domains are given respectively by

$$
\int d \Lambda=\int d \mathcal{V} \int d \mathcal{W} \quad d \mathcal{V}=d^{3} x \quad d \mathcal{W}=2 \pi m^{-1} d z d w B_{\|}^{*}
$$

hence identifying $B_{\|}^{*}$ as the variable part of the velocity space volume element. 
The Euler-Lagrange equations for $\phi$ are found by varying the Lagrangian with respect to $\phi$, yielding an integral over $d \mathcal{V}$ of $\delta \phi(\mathbf{x})$ times a coefficient, which is required to vanish. This produces

$$
\sum_{\mathrm{sp}} \int d \mathcal{W}\left[e J_{0} f+J_{0}\left(\mathcal{M} J_{0} \phi\right)-\left(J_{0} \mathcal{M}\right) \phi\right]=0
$$

where $\mathcal{M}$ is the polarisability given by

$$
\mathcal{M}=-\frac{e^{2}}{B} \frac{\partial f}{\partial w}
$$

It is customary in most treatments to linearise the polarisation term involving $\mathcal{M}$. This corresponds to replacing $f$ by a Maxwellian background $F^{M}$ in the term in the system Lagrangian which is due to the gyroscreening potential. Since the dependence of $F^{M}$ upon $w$ is proportional to $\exp (-w B / T)$, the polarisability is replaced by

$$
\mathcal{M} \quad \rightarrow \quad e^{2} F^{M} / T
$$

The Hamiltonian is replaced by

$$
H \quad \rightarrow \quad m \frac{z^{2}}{2}+w B+e J_{0} \phi
$$

and the polarisation equation is replaced by

$$
\sum_{\mathrm{sp}} \int d \mathcal{W}\left[e J_{0} f+e^{2} J_{0}\left(\frac{F^{M}}{T} J_{0} \phi\right)-\left(J_{0} \frac{F^{M}}{T}\right) \phi\right]=0
$$

where now only the source term depends on the variable $f$. The facts that $H$ is linear in $\phi$ and polarisation is linear in $f$ lead to the fact that this particular set of terms remains intact when the delta-f approximations are taken. Ultimately, mutual field/particle energy conservation depends on the properties of these terms, and the gyrofluid energy considerations descend directly from the total-f gyrokinetic ones.

Most treatments neglect the action of $J_{0}$ upon $F^{M}$, leaving the familiar form with $\left(e^{2} / T\right) F^{M}\left(J_{0}^{2}-1\right) \phi$, whose velocity space integration produces $\left(n e^{2} / T\right)\left(\Gamma_{0}-1\right) \phi$, where $\Gamma_{0}$ is an operator whose form in wavenumber space is multiplication of Fourier coefficients by $I_{0}(b) e^{-b}$ with argument $b=k_{\perp}^{2} \rho_{i}^{2}$ evaluated with the thermal gyroradius $\rho_{i}$, in turn given by $\rho_{i}^{2}=M T c^{2} / e^{2} B^{2}$ for species $i$ [7]. In recent total-f gyrokinetic computations, this operator is further simplified by using $\nabla \cdot\left(n M c^{2} / B^{2}\right) \nabla \phi$, its low- $k_{\perp}$ limit [31 33]. In these forms $n$ and $T$ are background constants or, at most, radial (across magnetic flux surfaces) profiles for the species density and temperature. 
When polarisation is linearised, the corresponding term in the Lagrangian becomes a field energy term, with $f$ replaced by $F^{M}$. Hence the second order field piece (gyroscreening potential) is moved from $H$ into the field energy term in the Lagrangian. Consistency is maintained only when these steps are taken together: if polarisation is linear then the gyroscreening potential must be absent in $H$, or vice versa [16].

The equation for $f$ is now determined by the particle equations of motion with the linearised $H$, with the actual form remaining intact. It is given by

$$
B_{\|}^{*} \frac{\partial f}{\partial t}+\nabla H \cdot \frac{c}{e} \frac{\mathbf{F}}{B} \cdot \nabla f+\frac{1}{m} \mathbf{B}^{*} \cdot\left(\frac{\partial H}{\partial z} \nabla f-\frac{\partial f}{\partial z} \nabla H\right)=0
$$

Eqs. (13]14]15) form the electrostatic version of the total-f gyrokinetic model which is our starting point for the rest of the derivation. First, the simplest extension to include shear Alfvén dynamics is given, then the delta-f model and its energy theorem are presented, and then the gyrofluid model descends from that.

\section{A. Electromagnetic extension}

The discussion of the electromagnetic version of the model is kept brief because the point is not to discuss various representations but to establish what is needed to proceed to the delta-f formulation. This version retains $z$ as the parallel velocity coordinate, not the canonical parallel momentum (cf. Ref. [34]). The particle Lagrangian $L_{p}$ retains the form in Eq. (1) with $\mathbf{A}^{*}$ now expanded to include the parallel magnetic potential, $A_{\|}$, only, so that

$$
\mathbf{A}^{*}=\mathbf{A}+\left(J_{0} A_{\|}+m z \frac{c}{e}\right) \mathbf{b}
$$

The main complications are that the part multiplying $\mathbf{b}$ is not only spatially variable but contains a time dependent field variable. The particle equations of motion become

$$
B_{\|}^{*} \frac{d \mathbf{x}}{d t}=z \mathbf{B}^{*}-\frac{c}{e B} \mathbf{F} \cdot \nabla H \quad m B_{\|}^{*} \frac{d z}{d t}=-\mathbf{B}^{*} \cdot \nabla H-\frac{e}{c} B_{\|}^{*} J_{0} \frac{\partial A_{\|}}{\partial t}
$$

with $\mathbf{B}^{*}$ now containing magnetic nonlinearities involving $\nabla\left(J_{0} A_{\|}\right)$. The overall Lagrangian including the fields becomes

$$
L=\sum_{\mathrm{sp}} \int d \Lambda f\left[\frac{e}{c} \mathbf{A}^{*} \cdot \frac{d \mathbf{x}}{d t}+w \frac{m c}{e} \frac{d \theta}{d t}-H\right]-\int d \mathcal{V} \frac{1}{8 \pi}\left|\nabla_{\perp} A_{\|}\right|^{2}
$$

with the last term representing the magnetic energy, and with $A_{\|}$also occurring in $\mathbf{A}^{*}$. Variation of this with respect to $\phi$ is unchanged, and produces Eq. (10) or its simplifications 
such as Eq. (14). Variation with respect to $A_{\|}$produces the induction equation (gyrokinetic version of Ampere's law),

$$
\nabla_{\perp}^{2} A_{\|}=-\frac{4 \pi}{c} \sum_{\mathrm{sp}} \int d \mathcal{W}\left[e J_{0} z f\right]
$$

where the term on the right gives the current, and without using the canonical parallel momentum as a coordinate there is no complication with the skin depth. The latter does however remain implicitly present as the electromagnetic gyrokinetic Vlasov equation for this Lagrangian,

$$
B_{\|}^{*} \frac{\partial f}{\partial t}-\frac{e}{m c} B_{\|}^{*} \frac{\partial f}{\partial z} J_{0} \frac{\partial A_{\|}}{\partial t}+\nabla H \cdot \frac{c}{e} \frac{\mathbf{F}}{B} \cdot \nabla f+\frac{1}{m} \mathbf{B}^{*} \cdot\left(\frac{\partial H}{\partial z} \nabla f-\frac{\partial f}{\partial z} \nabla H\right)=0
$$

now includes the time dependent induction effect through the explicit time derivative of $A_{\|}$.

\section{B. The delta-f approximations}

The delta-f Vlasov equation results from the expansion $f=F^{M}+\delta f$, with $\delta f$ a correction to a Maxwellian background $F^{M}$ whose sole spatial dependence is through the magnetic field strength $B$, and application of the delta-f ordering. Prior to the application of strict deltaf ordering, the parallel dynamics is linearised, so that the only nonlinear terms are those resulting from $\nabla H \cdot \mathbf{F} \cdot \nabla$ or $\mathbf{B}^{*} \cdot \nabla$, arising from the perturbed contributions of $H, A_{\|}$and $f$. With $\rho_{i} / L$ ordered small (with $L$ any background scale), $B_{\|}^{*}$ is replaced by $B$. Further, the curvature is set such that $\mathbf{b} \cdot \nabla \mathbf{b}=\nabla_{\perp} \log B$. This yields

$$
\begin{gathered}
B \frac{\partial g}{\partial t}+\nabla \psi_{e} \cdot \frac{c}{B} \mathbf{F} \cdot \nabla(\delta f)+\frac{m z^{2}+w B}{e} \nabla \log B \cdot \frac{c}{B} \mathbf{F} \cdot \nabla h \\
+\frac{1}{m} \frac{\partial H_{0}}{\partial z} \mathbf{B} \cdot \nabla h-\frac{1}{m} \frac{\partial h}{\partial z} \mathbf{B} \cdot \nabla H_{0}=0
\end{gathered}
$$

where the auxiliary variables are

$$
g=\delta f+\frac{F^{M}}{T} e \frac{z}{c} J_{0} A_{\|} \quad h=\delta f+\frac{F^{M}}{T} e J_{0} \phi \quad \psi_{e}=J_{0}\left(\phi-\frac{z}{c} A_{\|}\right)
$$

These are the inductive response, nonadiabatic response, and gyrokinetic potential, respectively. The zeroth order Hamiltonian and the Maxwellian are given by

$$
H_{0}=m \frac{z^{2}}{2}+w B \quad F^{M}=n(2 \pi T / m)^{-3 / 2} \exp \left(-H_{0} / T\right)
$$

with $m$ and $e$ and $n$ and $T$ the species background parameters. 
The terms in Eq. (21) are referred to as nonlinear advection, magnetic drift, and parallel trapping and streaming, respectively. Nonlinear advection is by the perturbed Hamiltonian, which includes ExB advection and the magnetic flutter nonlinearities, through $\phi$ and $A_{\|}$, respectively. The grad-B and curvature drifts are combined, and departures from an inverse major radius dependence of $B$ are neglected. The parallel dynamics includes both streaming $\left(\partial H_{0} / \partial z\right)$ and magnetic trapping $\left(\nabla H_{0}=w B \nabla \log B\right)$. The parallel dynamics is linearised, with both static and inductive pieces of $E_{\|}$appearing with $(m z / T) F^{M}$, leading to the expressions $h$ and $g$, respectively.

Now the strict form of delta-f ordering is applied. This refers to the split between the perpendicular and parallel coordinate directions following from the $k_{\|} \ll k_{\perp}$ ordering. A field aligned coordinate system is assumed, with $x$ and $y$ the perpendicular coordinates respectively following the radial and electron drift directions, and the coordinate $s$ following the parallel direction. This is done for a general tokamak geometry following Refs. [35 37]. Axisymmetry renders $\partial / \partial y=0$ for the background magnetic field $\mathbf{B}$. The unperturbed parallel gradient is given by

$$
\mathbf{B} \cdot \nabla=B^{s} \frac{\partial}{\partial s} \quad \text { hence } \quad \frac{\partial}{\partial x} \sim \frac{\partial}{\partial y} \gg \frac{\partial}{\partial s}
$$

Under this ordering we have $B=B(s)$, and hence $B$ and $F^{M}$ are independent of $x$ and $y$. However, $\log B$ in the magnetic drift terms must be re-formed in order to keep both the interchange $(\partial \log B / \partial x)$ and geodesic $(\partial \log B / \partial s)$ curvature contributions. The derivatives of $\log B$ are evaluated, and then the resulting form is restricted to have dependence upon $s$ only as with the rest of the geometry. This introduces the curvature operator $\mathcal{K}$, which is expressed in terms of a vector-gradient contraction,

$$
\mathcal{K} \equiv \mathcal{K}^{x}(s) \frac{\partial}{\partial x}+\mathcal{K}^{y}(s) \frac{\partial}{\partial y}
$$

where the components are given by

$$
\mathcal{K}^{\{x, y\}}=-\nabla \log B^{2} \cdot \frac{c \mathbf{F}}{B^{2}} \cdot \nabla\{x, y\}
$$

It is necessary for free energy conservation that $\mathcal{K}$ be a pure divergence. This is guaranteed by maintaining

$$
\frac{\partial}{\partial x} \mathcal{K}^{x}+\frac{\partial}{\partial y} \mathcal{K}^{y}=0
$$


which is trivially satisfied by having the $\mathcal{K}^{a}$ components depend on $s$ only. Correspondence to the linear forms in Refs. [1, 2] is to identify $\mathcal{K}$ with $-2 i \omega_{d}$, where $\omega_{d}$ is the toroidal drift frequency.

After this, the derivatives in the nonlinear advection term are restricted to $\{x, y\}$ only, and the trapping/streaming terms involve derivatives in $\{s, z\}$ only. The resulting delta-f ordered gyrokinetic equation is

$$
\frac{\partial g}{\partial t}+\frac{c}{B_{0}}\left[\left(J_{0} \psi_{e}\right), h\right]_{x y}-\frac{m z^{2}+w B}{2 e} \mathcal{K}(h)+\frac{B^{s}}{m B}\left[H_{0}, h\right]_{z s}=0
$$

where the brackets are given by

$$
[f, g]_{a b}=\frac{\partial f}{\partial x^{a}} \frac{\partial g}{\partial x^{b}}-\frac{\partial f}{\partial x^{b}} \frac{\partial g}{\partial x^{a}}
$$

with $a$ and $b$ denoting two of the phase space coordinates, $\mathcal{K}$ is the magnetic curvature operator. The coefficients $c, m, e$, and $B_{0}$ are constants; $x, y, s, z$, and $w$ are coordinates; and $B$, the metric coefficients contained in $J_{0}$, and the components of $\mathcal{K}$ are functions of $s$ only. The Maxwellian $F^{M}$ is a function of $B, z$, and $w$ only. Field aligned Hamada coordinates constructed as in Refs. [36, 37] are assumed. Hence, $B^{s}$ and $F^{x y} / B^{2}$ are flux functions, which in the local limit reduce to constants. Following this is the use of the constant $B_{0}$, the average value of $B$ on a flux surface.

The field variables $\phi$ and $A_{\|}$are functions of $\mathbf{x}$ and are determined in the total-f theory by the polarisation and induction equations, respectively. In the total-f theory these equations are found by variation of the total action with respect to the field variables. The delta-f forms are the corresponding linearised versions. The polarisation equation is

$$
\sum_{\mathrm{sp}} \int d \mathcal{W}\left[e J_{0} g+e^{2} \frac{F^{M}}{T}\left(J_{0}^{2}-1\right) \phi\right]=0
$$

resulting from Eq. (14). The induction equation is

$$
\nabla_{\perp}^{2} A_{\|}+\sum_{\mathrm{sp}} \frac{4 \pi}{c} \int d \mathcal{W}\left[e z J_{0} g-\frac{e^{2}}{c} z^{2} \frac{F^{M}}{T} J_{0}^{2} A_{\|}\right]=0
$$

resulting from Eq. (19). In each case the delta-f form of the velocity space integral $\int d \mathcal{W}$ has reduced to

$$
d \mathcal{W}=2 \pi m^{-1} d z d w B
$$

The source terms in these equations follow directly from the lowest order interaction Lagrangian terms,

$$
\int d \Lambda(\delta f)\left(-e J_{0} \phi\right) \quad \int d \Lambda(\delta f) \frac{z}{c}\left(e J_{0} A_{\|}\right)
$$


respectively, where we can replace $f$ by $\delta f$ because these terms are linear in $f$. The Hermitian property of $J_{0}$ and its commutation with $z$ plays the central role. This is why the way $J_{0}$ mixes moments through its $w$-dependence must be established the same way for the field variable derivatives in the Vlasov equation and the moment source terms in the polarisation and induction equations. The interchangeable use of $g$ or $\delta f$ in Eq. (30) follows from the antisymmetry of $z J_{0}$ in velocity space.

\section{Energy and free energy in the gyrokinetic models}

The conserved energy $\mathcal{E}$ for the total-f model is given by application of the Noether theorem to its Lagrangian [16, 17]. With $H$ and $L$ given in Eqs. (38), the energy is merely the phase space integral of $H$ over $f$,

$$
\mathcal{E}=\sum_{\mathrm{sp}} \int d \Lambda H f=\sum_{\mathrm{sp}} \int d \Lambda\left\{H_{0}+e J_{0} \phi-\frac{e^{2}}{2 B} \frac{\partial}{\partial w}\left[J_{0}\left(\phi^{2}\right)-\left(J_{0} \phi\right)^{2}\right]\right\} f
$$

The polarisation equation (Eq. 10) may be used to recast this in terms of

$$
\mathcal{E}=\sum_{\mathrm{sp}} \int d \Lambda\left\{H_{0} f+\frac{\mathcal{M}}{2}\left[J_{0}\left(\phi^{2}\right)-\left(J_{0} \phi\right)^{2}\right]\right\}
$$

where the combination involving $\phi$ is the generalised ExB kinetic energy (in the low- $k_{\perp}$ limit it reduces to the familiar form in terms of $v_{E}^{2}$, i.e., the usual ExB velocity squared). Note the use of the Hermitian property of $J_{0}$ as well as integration by parts of $\partial / \partial w$.

In the form with linearised polarisation, the energy is equivalently given by Eq. (34) with $f \rightarrow F^{M}$ in the gyroscreening term. Again the polarisation equation (here, Eq. 14) is used to eliminate the $f e J_{0} \phi$ term, to find

$$
\mathcal{E}=\sum_{\mathrm{sp}} \int d \Lambda\left[H_{0} f+e^{2} \frac{F^{M}}{T}\left(1-J_{0}^{2}\right) \frac{\phi^{2}}{2}\right]
$$

with $\mathcal{M}=\left(e^{2} / T\right) F^{M}$ inserted explicitly and the action of $J_{0}$ upon $F^{M} / T$ neglected. The notation $J_{0}^{2} \phi^{2}$ is shorthand for $\left(J_{0} \phi\right)^{2}$. The velocity space integral over $F^{M}$ can be done explicitly to find

$$
\mathcal{E}=\sum_{\mathrm{sp}} \int d \Lambda\left[H_{0} f+n \frac{e^{2}}{T}\left(1-\Gamma_{0}\right) \frac{\phi^{2}}{2}\right]
$$

where again $\Gamma_{0} \phi^{2}$ is shorthand for $\phi\left(\Gamma_{0} \phi\right)$. 
This field energy term is the same in the delta-f model. The interaction Lagrangian term (implicit in the delta-f model) is also the same, due to the linearity in both $f$ and $\phi$. This fact was used to motivate the discussion of free energy in delta-f models generally [20, 25, 26]. Here, however, we have an even easier path, noticing that every term in Eq. (28) is a bracket with $h$, i.e., a first order derivative on $h$ and also a total divergence. It is trivial to multiply Eq. (28) by $h$ and any other quantity which commutes with all the brackets to obtain conserved quantities, called Casimirs of the system. The one identified with free energy is quadratic in $h$ and must have dimensions of $n T$ integrated over space. Hence the factor $\left(T / F^{M}\right)$ which works since $F^{M}$ depends on $\{s z w\}$ only and commutes with the $z s$-bracket, and $T$ is constant. The contribution quadratic in $\delta f$ is much like a smallamplitude form of the thermodynamic entropy, while the contributions due to $\phi$ and $A_{\|}$ become equivalent to the ExB and magnetic field energies present in the total-f model, once the polarisation and induction equations are used. Details and underlying considerations are in Ref. [25].

The energy theorem for these equations (28,30,31) is given by

$$
\frac{\partial \mathcal{E}}{\partial t}=\frac{\partial}{\partial t} \sum_{\mathrm{sp}} \int d \Lambda\left[\frac{T}{F^{M}} \frac{h g}{2}\right]
$$

or equivalently,

$$
\frac{\partial \mathcal{E}}{\partial t}=\frac{\partial}{\partial t} \sum_{\mathrm{sp}} \int d \Lambda\left[\frac{T}{F^{M}} \frac{(\delta f)^{2}}{2}+e^{2} \frac{F^{M}}{T}\left(1-J_{0}^{2}\right) \frac{\phi^{2}}{2}\right]+\frac{\partial}{\partial t} \int d \mathcal{V} \frac{1}{8 \pi}\left|\nabla_{\perp} A_{\|}\right|^{2}
$$

reflecting mutual conservation of thermal and kinetic free energy, the E-cross-B energy, and the magnetic fluctuation energy, each given by

$$
\begin{gathered}
\mathcal{E}_{f}=\sum_{\mathrm{sp}} \int d \Lambda \frac{T}{F^{M}} \frac{(\delta f)^{2}}{2} \\
\mathcal{E}_{E}=\sum_{\mathrm{Sp}} \int d \Lambda e^{2} \frac{F^{M}}{T}\left(1-J_{0}^{2}\right) \frac{\phi^{2}}{2} \\
\mathcal{E}_{M}=\int d \mathcal{V} \frac{1}{8 \pi}\left|\nabla_{\perp} A_{\|}\right|^{2}
\end{gathered}
$$

respectively.

The need to use the field equations to bring the factor of $h$ under the $\partial / \partial t$ in Eq. (38) is the reason the choice of second-order Casimir to identify as the total free energy is unique. Any integrable function of $h$ multiplying Eq. (28) will produce a vanishing phase space 
integral. However, the operation through Eqs. (30|31) will result in a single time derivative only if the multiplier is $\left(T / F^{M}\right) h$. This yields the identification of $\left(T / F^{M}\right) h g / 2$ as the free energy density and therefore $\mathcal{E}$ given by Eq. (39) as the total free energy.

\section{THE GYROFLUID MOMENT SET AND FREE ENERGY}

The basic prescription of a gyrofluid model is the set of moments kept as dependent variables. The simplest version uses the density only [6]. One level up from that is a three dimensional version using densities and parallel velocities, equivalent in scope to the familiar four field fluid models [14]. With the temperature gradient of either species setting the basic dynamical character, at least four moments, one each for the density $\tilde{n}$, parallel velocity $\widetilde{u}_{\|}$, and parallel and perpendicular temperature $\widetilde{T}_{\|}$and $\widetilde{T}_{\perp}$, are needed [2]. These temperatures are given by the parallel and perpendicular energy moments (over $m z^{2} / 2$ and $w B$, respectively) divided by the density. The perp/parallel separation in the temperatures is made necessary by the underlying dynamics: only $\widetilde{T}_{\|}$enters parallel (hence Alfvén) dynamics, and only $\widetilde{T}_{\perp}$ is involved in polarisation (through the $w B$ dependence of $J_{0}$ ). In the nonlinear dynamics there is moment mixing between $\widetilde{n}$ and $\widetilde{T}_{\perp}$ but not with $\widetilde{T}_{\|}$, so even in the absence of curvature and grad-B drifts the responses of $\widetilde{T}_{\perp}$ and $\widetilde{T}_{\|}$to the rest of the dynamics are different. Finally, magnetised plasma turbulence takes place at time scales for which the parallel sound wave transit frequency $c_{s} / q R$ is very slow. Both the parallel viscosity and the perp/parallel components of the parallel heat flux have time-dependent responses to velocity and temperature gradients. In a gyrofluid model the parallel viscosity is proportional to the difference $\widetilde{T}_{\|}-\widetilde{T}_{\perp}$, which is already taken care of as $\widetilde{T}_{\|}$and $\widetilde{T}_{\perp}$ have their own time dependent equations. This means that the perp/parallel components of the parallel heat flux $\left(\widetilde{q}_{\|\| \|}\right.$and $\widetilde{q}_{\perp \|}$, moments over $m z^{3} / 2$ and $z w B$, respectively) also require their own time dependent equations. The six moment models [1, 2, 4, 15] are the result.

Here and below, the tilde symbols are used to distinguish the dependent variables from the parameters. There is one set of moment variables per species, with all species contributing to polarisation and induction and the field energy pieces. 
The moment variable list is given by

$$
\begin{array}{cc}
\widetilde{n}=\int d \mathcal{W}(\delta f) & n \widetilde{T}_{\perp}=\int d \mathcal{W}(w B-T)(\delta f) \\
n \widetilde{u}_{\|}=\int d \mathcal{W} z(\delta f) & \widetilde{q}_{\|}=\int d \mathcal{W}\left(m z^{2}-3 T\right) \frac{z}{2}(\delta f) \\
n \widetilde{T}_{\|}=\int d \mathcal{W}\left(m z^{2}-T\right)(\delta f) & \widetilde{q}_{\perp}=\int d \mathcal{W}(w B-T) z(\delta f)
\end{array}
$$

where here and below $\widetilde{q}_{\|}$and $\widetilde{q}_{\perp}$ are used as shorthand for $\widetilde{q}_{\|\|}$and $\widetilde{q}_{\perp \|}$, respectively. Heat fluxes perpendicular to $\mathbf{B}$ are given by the curvature and grad-B drifts, as combinations of $\widetilde{n}, \widetilde{T}_{\|}$, and $\widetilde{T}_{\perp}$, and therefore are not written explicitly.

Use of a finite set of moment variables to represent $\delta f$ implies a representation of $\delta f$ in terms of a finite-degree polynomial in velocity space with those same moment variables as coefficients [26]. The Hermite polynomial decomposition of the distribution function in terms of the set of moment variables in Eqs. (43) is given by

$$
\begin{aligned}
\delta f=F^{M}\left[\frac{\widetilde{n}}{n}+\frac{\widetilde{u}_{\|}}{V} \frac{z}{V}\right. & +\frac{1}{2} \frac{\widetilde{T}_{\|}}{T}\left(\frac{z^{2}}{V^{2}}-1\right)+\frac{\widetilde{T}_{\perp}}{T}\left(\frac{w B}{m V^{2}}-1\right) \\
& \left.+\frac{1}{3} \frac{\widetilde{q}_{\|}}{n T V}\left(\frac{z^{2}}{V^{2}}-3\right) \frac{z}{V}+\frac{\widetilde{q}_{\perp}}{n T V}\left(\frac{w B}{m V^{2}}-1\right) \frac{z}{V}\right]
\end{aligned}
$$

where $V$ is the species thermal velocity given by $V^{2}=T / m$. The coefficients are chosen for orthogonality and to recover the above definitions of the moment variables. Insertion of this form into the portion of the delta-f free energy in Eq. (39) dependent on $\delta f$ yields the gyrofluid free energy. The part dependent on the state variables, $\widetilde{n}$ or $\widetilde{T}_{\|}$or $\widetilde{T}_{\perp}$, is the thermal free energy, and the part dependent on the flux variables, $\widetilde{u}_{\|}$or $\widetilde{q}_{\|}$or $\widetilde{q}_{\perp}$, is the kinetic free energy. The spatial density of the thermal free energy is

$$
U_{t}=\frac{n T}{2}\left[\left(\frac{\tilde{n}}{n}\right)^{2}+\frac{1}{2}\left(\frac{\widetilde{T}_{\|}}{T}\right)^{2}+\left(\frac{\widetilde{T}_{\perp}}{T}\right)^{2}\right]
$$

and the density of the kinetic free energy is

$$
U_{v}=\frac{n T}{2}\left[\left(\frac{\widetilde{u}_{\|}}{V}\right)^{2}+\frac{2}{3}\left(\frac{\widetilde{q}_{\|}}{n T V}\right)^{2}+\left(\frac{\widetilde{q}_{\perp}}{n T V}\right)^{2}\right]
$$

Together, $U_{t}+U_{v}$ represent the delta-f thermal free energy given by $\mathcal{E}_{f}$ in Eq. (40), and are derived directly from it. 
The field energy pieces are the same as in the delta-f gyrokinetic version. Evaluating the integral over $\left(1-J_{0}^{2}\right) F^{M}$ in Eq. (41), the gyrofluid E-cross-B energy density is found. The magnetic energy density carries over directly from Eq. (42). These are given by

$$
U_{E}=\sum_{\mathrm{sp}}\left[n e^{2} \frac{\left(1-\Gamma_{0}\right)}{T} \frac{\phi^{2}}{2}\right] \quad U_{M}=\frac{1}{8 \pi}\left|\nabla_{\perp} A_{\|}\right|^{2}
$$

respectively. The operator $\Gamma_{0}$ reflects gyroscreening. As noted above, its form in wavenumber space is the function

$$
\Gamma_{0}(b) \equiv I_{0}(b) e^{-b} \quad \text { with } \quad b=k_{\perp}^{2} \rho^{2}
$$

where $\rho=V / \Omega$ is the thermal gyroradius. Recall that in the local model $\Gamma_{0}$ involves derivatives with respect to $x$ and $y$ only, while $B=B(s)$ so that the Hermitian property is maintained. If wavenumber space is unavailable, the Padé approximant $\Gamma_{0}(b)=(1+b)^{-1}$ is used. Eqs. (45-47) are the same as in Ref. [15], but now they have a firm derivation in terms of the delta-f gyrokinetic version, applying the procedure of Ref. [26] to the result in Eq. (39)).

\section{CLOSURE RULES FOR GYROAVERAGING}

The evaluation of $J_{0}^{2}$ in polarisation was trivial because it is integrated over $F^{M}$ and the integral of $J_{0}(b)^{2} e^{-b}$ is well known. However, in the source term in polarisation (Eq. 30) only one factor of $J_{0}$ appears. A closure approximation for $\int d \mathcal{W} J_{0} F^{M}$ is needed. The one used previously was decided from the properties of resulting linear eigenfunctions [1, 2]. However it is possible to evaluate this directly. Taking the density moment over $h$, we find

$$
\int d \mathcal{W} h=\widetilde{n}+\int d \mathcal{W} \frac{F^{M}}{T} e J_{0} \phi
$$

In the second term, $\phi$ is dependent on space only, and the fact that under the delta-f model $F^{M}$ commutes with perpendicular spatial derivatives may be used to do the velocity space integral separately. This moment defines the basic gyroaveraging operator,

$$
\Gamma_{1} \equiv \frac{1}{n} \int d \mathcal{W} F^{M} J_{0}
$$

which acts the same way on any spatially dependent moment or field variable. It is merely a special function, as we find by inserting the form of $F^{M}$, doing the integral over $z$, and defining $x=w B / T$,

$$
\Gamma_{1}(b)=\int_{0}^{\infty} d x e^{-x} J_{0}(\sqrt{2 b x})
$$


This form was used by Knorr et al [6]. Conforming to the practice of Beer et al [1], however, we keep to the definition

$$
\Gamma_{1}(b) \rightarrow \Gamma_{0}^{1 / 2}(b)
$$

and hence the operation of $\Gamma_{1}$ on a spatial variable is like that of $\Gamma_{0}$ in wavenumber space, with $\Gamma_{0}^{1 / 2}(b)$ is used rather than $\Gamma_{0}(b)$. If wavenumber space is unavailable, the Padé approximant $\Gamma_{0}(b)=(1+b / 2)^{-1}$ is used. The label $\Gamma_{1}$ is used for generality: any Hermitian operator is admissible if consistency is the only requirement, so that if $F^{M}$ is non-Maxwellian then some form other than $\Gamma_{0}^{1 / 2}$ is chosen. These considerations are explained and justified in Ref. [1].

In the polarisation equation the source term is $\int d \mathcal{W} e J_{0}(\delta f)$, which contains moments over both unity and $w B$ times $F^{M}$. The corresponding closure approximation for this is found by inserting Eqs. (44) into the factor of $e J_{0}(\delta f)$ in Eq. (30) to obtain

$$
\int d \mathcal{W} e J_{0}(\delta f)=n e\left(\Gamma_{1} \frac{\widetilde{n}}{n}+\Gamma_{2} \frac{\widetilde{T}_{\perp}}{T}\right)
$$

where $\Gamma_{2}$ is given by

$$
\Gamma_{2}=\int d \mathcal{W} \frac{w B-T}{T} F^{M} J_{0}=T \frac{\partial}{\partial T} \int d \mathcal{W} F^{M} J_{0}
$$

following both appearances of $T$ in the factors in $F^{M}$ which remain after the integral over $z$ is done (cf. Eq. 23). It follows that whichever approximation is taken for $\Gamma_{1}$ we always have

$$
\Gamma_{2}=T \frac{\partial \Gamma_{1}}{\partial T} \rightarrow b \frac{\partial \Gamma_{1}}{\partial b}
$$

where the latter form is the one to use in wavenumber space.

We refer to $\Gamma_{1}$ and $\Gamma_{2}$ as the first and second gyroaveraging operators, or alternatively as the gyroaverging operator and its first FLR correction, respectively. For a six-moment model, this is as far as the moment hierarchy goes. If the moment over $(w B)^{2}$ is a dependent variable in an extended version of the theory, then there is a further third gyroaveraging operator involving second derivatives of $\Gamma_{1}$ with respect to $b$, and so forth. Strictly speaking, the $(w B)^{2}$ moment will result in a form determined by $\partial^{2} \Gamma_{1} / \partial(\log b)^{2}$. However, in this case we have to replace this 4 th moment with a form mandated by energy conservation.

Energy conservation in the delta-f Vlasov equation works with $h$ appearing under all the derivatives and free energy evolution determined by multiplication of the equation by $h$ 
and integrating over phase space with sum over species (Eqs. 28, 38, 39). The corresponding form of this is that the moments of $h$ appear under the derivatives in the gyrofluid moment equations. These moments are merely those over $\delta f$ together with contributions due to $J_{0} \phi$. Only two of these contributions are nonzero, as the others vanish due to odd symmetry or orthogonality in the integrals over $z$. The surviving ones are

$$
\int d \mathcal{W} h=\tilde{n}+n \frac{e}{T} \Gamma_{1} \phi \quad \quad \int d \mathcal{W} \frac{w B-T}{T} h=n \frac{\widetilde{T}_{\perp}}{T}+n \frac{e}{T} \Gamma_{2} \phi
$$

Hence in the linear terms in the moment equations, derivatives of $\tilde{n}+n(e / T) \Gamma_{1} \phi$ must appear in that combination, and the same holds for $\widetilde{T}_{\perp}+e \Gamma_{2} \phi$. This was the procedure by which Ref. [15] repaired gyrofluid energy conservation. The higher moments (4th and 5th) over $h$ which occur in the equations are then determined by the same ones over $\delta f$, which are straightforward. Hence for the $(w B)^{2}$ moment we have

$$
\int d \mathcal{W}(w B)^{2} h=\int d \mathcal{W}(w B)^{2}(\delta f)+\frac{e}{T}\left(\int d \mathcal{W} w B w B F^{M} J_{0}\right) \phi
$$

The first piece is found by straightforward evaluation

$$
\int d \mathcal{W}(w B)^{2}(\delta f)=2 p T\left(\frac{\widetilde{n}}{n}+2 \frac{\widetilde{T}_{\perp}}{T}\right)
$$

Hence this moment over $h$ must have the form found by combining $\Gamma_{1} \phi$ with $\widetilde{n}$ and $\Gamma_{2} \phi$ with $\widetilde{T}_{\perp}$

$$
\int d \mathcal{W}(w B)^{2} h=2 p T\left[\left(\frac{\widetilde{n}}{n}+\frac{e}{T} \Gamma_{1} \phi\right)+2\left(\frac{\widetilde{T}_{\perp}}{T}+\frac{e}{T} \Gamma_{2} \phi\right)\right]
$$

Subtracting these two we find the required form,

$$
\frac{e}{T}\left(\int d \mathcal{W} w B w B F^{M} J_{0}\right) \phi=2 p T \frac{e}{T}\left(\Gamma_{1}+2 \Gamma_{2}\right) \phi
$$

and hence the requirement on the operators

$$
\frac{1}{n} \int d \mathcal{W}\left(\frac{w B}{T}\right)^{2} F^{M} J_{0}=2\left(\Gamma_{1}+2 \Gamma_{2}\right)
$$

and also

$$
\frac{1}{n} \int d \mathcal{W}\left(\frac{w B-T}{T}\right)^{2} F^{M} J_{0}=\left(\Gamma_{1}+2 \Gamma_{2}\right)
$$

This replaces the form which would be found from two derivatives applied to $\Gamma_{1}$. But that third form is only viable in the case the 4 th moments of $\delta f$ are retained as dynamical variables. In the six-moment model however the last equation above replaces that, and the 
moment hierarchy is closed. The closure of higher moments over $\delta f$ is given by its form in Eq. (44), and then the higher moments over $J_{0}$ are given by the requirement that the combinations in Eq. (56) always appear intact in the moments over $h$.

Equivalently, we list the two approximations involved in the gyrofluid model: (1) the choice of moments to keep as dynamical variables, and (2) the form taken for $\Gamma_{1}$. The rest

of the model then follows by simple evaluation constrained by energy conservation (keeping the pieces of moments over $h$ together).

\section{GYROFLUID POLARISATION AND RELATION TO ENERGY CONSERVA- TION}

We now return to the gyrofluid polarisation equation. Insertion of $\delta f$ from Eq. (44) into Eq. (30) and using the definitions of $\Gamma_{1}$ and $\Gamma_{2}$ in Eqs. (50,54), we have

$$
\sum_{\mathrm{sp}}\left[n e\left(\Gamma_{1} \frac{\tilde{n}}{n}+\Gamma_{2} \frac{\widetilde{T}_{\perp}}{T}\right)+n e^{2} \frac{\Gamma_{0}-1}{T} \phi\right]=0
$$

As in the delta-f gyrokinetic polarisation equation (Eq. 30), this is a statement of strict quasineutrality, with each species charge density given by the gyrocenter part (moment variables) and the polarisation part (due to the electrostatic potential).

In the total-f or delta-f gyrokinetic models, we can recover the interaction Lagrangian in Eq. (33) by multiplying the polarisation equation by $\phi$ and integrating over space (essentially un-doing the steps by which the polarisation equation is derived in the first place). Under linearised polarisation the interaction Lagrangian is the same in both the total- and delta-f models, since the term is linear in both $f$ and $\phi$. We can do the same in the gyrofluid version, either operation by $\int d \mathcal{V} \phi \times$ or simply by inserting $\delta f$ from Eq. (44) into Eq. (33). In all these forms, the Hermicity of $J_{0}$ and hence $\Gamma_{1}$ and $\Gamma_{2}$ has a central role.

For $\phi$ the result is

$$
L_{\text {int }, \phi}=-\sum_{\mathrm{sp}} \int d \mathcal{V}\left[e \phi\left(\Gamma_{1} \widetilde{n}+\frac{n}{T} \Gamma_{2} \widetilde{T}_{\perp}\right)\right]
$$

whose variation with respect to $\phi$ recovers the gyrocenter source terms in Eq. (63). Applying the Hermitian property of the $\Gamma$ 's, this is equivalent to

$$
L_{\mathrm{int}, \phi}=-\sum_{\mathrm{sp}} \int d \mathcal{V}\left[e\left(\widetilde{n} \phi_{G}+\frac{n}{T} \widetilde{T}_{\perp} \Omega_{G}\right)\right]
$$


where

$$
\phi_{G}=\Gamma_{1}(\phi)=\frac{1}{n} \int d \mathcal{W} F^{M}\left(J_{0} \phi\right) \quad \Omega_{G}=\Gamma_{2}(\phi)=\int d \mathcal{W} \frac{w B-T}{n T} F^{M}\left(J_{0} \phi\right)
$$

are defined as the first and second gyroaveraged potentials, respectively. This is the underlying reason $\phi_{G}$ is associated with $\widetilde{n}$ and $\Omega_{G}$ with $\widetilde{T}_{\perp}$ and, ultimately, why they must appear together in derivatives representing energy transfer processes between the various equations.

The corresponding forms for $A_{\|}$use the delta-f induction equation (Eq. 31) and the part of Eq. (33) due to $A_{\|}$, with the result

$$
L_{\mathrm{int}, A_{\|}}=\sum_{\mathrm{sp}} \int d \mathcal{V}\left[n \frac{e}{c} A_{\|}\left(\Gamma_{1} \widetilde{u}_{\|}+\Gamma_{2} \frac{\widetilde{q}_{\perp}}{T}\right)\right]
$$

Variation of this with respect to $A_{\|}$recovers the source term in the gyrofluid Ampere's law,

$$
\nabla_{\perp}^{2} A_{\|}+\frac{4 \pi}{c} \sum_{\mathrm{sp}}\left[n e\left(\Gamma_{1} \widetilde{u}_{\|}+\Gamma_{2} \frac{\widetilde{q}_{\perp}}{T}\right)\right]=0
$$

We can also find this by inserting the form for $\delta f$ in Eq. (44) into the gyrokinetic Ampere's law (Eq. 31) and evaluating the velocity space integrals. Using the Hermitian property of the $\Gamma$ 's, the electromagnetic interaction Lagrangian can also be re-cast as

$$
L_{\mathrm{int}, A_{\|}}=\sum_{\mathrm{sp}} \int d \mathcal{V}\left[n \frac{e}{c}\left(\widetilde{u}_{\|} A_{G}+\frac{\widetilde{q}_{\perp}}{T} \chi_{G}\right)\right]
$$

where

$$
A_{G}=\Gamma_{1}\left(A_{\|}\right)=\frac{1}{n} \int d \mathcal{W} F^{M}\left(J_{0} A_{\|}\right) \quad \chi_{G}=\Gamma_{2}\left(A_{\|}\right)=\int d \mathcal{W} \frac{w B-T}{n T} F^{M}\left(J_{0} A_{\|}\right)
$$

are defined as the first and second gyroaveraged magnetic potentials, respectively. This leads to the appearance of $\widetilde{u}_{\|}$and $A_{G}$ together, and $\widetilde{q}_{\perp}$ and $\chi_{G}$, under $\partial / \partial t$ in the gyrofluid moment equations.

All of this is closely related to conservation of free energy in terms of the functionals in Eqs. (38, 39) for the delta-f gyrokinetic model and Eqs. (45447) for the gyrofluid case. The field energy components in Eqs. (47) can be re-cast using the polarisation and induction equations in Eqs. (63,68) as

$$
\mathcal{E}_{E}=\sum_{\mathrm{sp}} \frac{1}{2} \int d \mathcal{V}\left[e\left(\phi_{G} \tilde{n}+n \Omega_{G} \frac{\tilde{T}_{\perp}}{T}\right)\right]
$$


and

$$
\mathcal{E}_{M}=\sum_{\mathrm{sp}} \frac{1}{2} \int d \mathcal{V}\left[\frac{e}{c}\left(n A_{G} \widetilde{u}_{\|}+\chi_{G} \frac{\widetilde{q}_{\perp}}{T}\right)\right]
$$

respectively. The time derivatives follow accordingly, and the time derivative of the total energy is given by

$$
\begin{array}{r}
\frac{\partial \mathcal{E}}{\partial t}=\sum_{\mathrm{sp}} \int d \mathcal{V}\left[\left(n e \phi_{G}+T \widetilde{n}\right) \frac{\partial}{\partial t} \frac{\widetilde{n}}{n}+n e \widetilde{u}_{\|} \frac{\partial}{\partial t}\left(\frac{1}{c} A_{G}+\frac{m}{e} \widetilde{u}_{\|}\right)+\frac{1}{2} \frac{n}{T} \widetilde{T}_{\|} \frac{\partial \widetilde{T}_{\|}}{\partial t}\right. \\
\left.+\frac{n}{T}\left(e \Omega_{G}+\widetilde{T}_{\perp}\right) \frac{\partial \widetilde{T}_{\perp}}{\partial t}+\frac{2}{3} \frac{m}{n T} \widetilde{q}_{\|} \frac{\partial}{\partial t} \frac{\widetilde{q}_{\|}}{n T}+e \frac{\widetilde{q}_{\perp}}{T} \frac{\partial}{\partial t}\left(\frac{1}{c} \chi_{G}+\frac{m}{e} \frac{\widetilde{q}_{\perp}}{n T}\right)\right]
\end{array}
$$

which is the direct correspondence to the $h(\partial g / \partial t)$ form in Eq. (39) for the delta-f model. This is the same energy equation as given in Ref. [15], but now it is a result, not a construction.

Following these we can find the moments over $h$ and $g$ defined in Eqs. (22) by inserting Eq. (44) for $\delta f$ and then straightforward evaluation of the velocity space integrals using the definitions in Eqs. (66/70) for the potentials. The moment list for $h$ in entirety is

$$
\begin{array}{cc}
\widetilde{n}+n \frac{e}{T} \phi_{G}=\int d \mathcal{W} h & n \widetilde{T}_{\perp}+n e \Omega_{G}=\int d \mathcal{W}(w B-T) h \\
n \widetilde{u}_{\|}=\int d \mathcal{W} z h & \widetilde{q}_{\|}=\int d \mathcal{W}\left(m z^{2}-3 T\right) \frac{z}{2} h \\
n \widetilde{T}_{\|}=\int d \mathcal{W}\left(m z^{2}-T\right) h & \widetilde{q}_{\perp}=\int d \mathcal{W}(w B-T) z h
\end{array}
$$

similar to Eqs. (43) as only $\widetilde{n}$ and $\widetilde{T}_{\perp}$ are affected. The moment list for $g$ in entirety is

$$
\begin{array}{cc}
\tilde{n}=\int d \mathcal{W} g & n \widetilde{T}_{\perp}=\int d \mathcal{W}(w B-T) g \\
n \widetilde{u}_{\|}+n \frac{e}{m c} A_{G}=\int d \mathcal{W} z g & \widetilde{q}_{\|}=\int d \mathcal{W}\left(m z^{2}-3 T\right) \frac{z}{2} g \\
n \widetilde{T}_{\|}=\int d \mathcal{W}\left(m z^{2}-T\right) g & \widetilde{q}_{\perp}+n T \frac{e}{m c} \chi_{G}=\int d \mathcal{W}(w B-T) z g
\end{array}
$$

where due to the extra factor of $z$ it is $\widetilde{u}_{\|}$and $\widetilde{q}_{\perp}$ which are affected. The moments over $g$ are the quantities appearing under $\partial / \partial t$ in the gyrofluid equations, while those over $h$ appear under the derivatives in the linear terms. 
The salient result of this section is the way the interaction Lagrangian and the Hermitian property fix the gyroaveraging operations for the potentials once they are decided for the moment variables, and vice versa. This relationship is what guarantees an energy conserving model using this particular procedure. And given these constraints, the free energy theorem follows naturally.

\section{THE GYROFLUID MOMENT EQUATIONS}

With the above in place, the straightforward derivation of the gyrofluid moment equations is essentially determined. The form of $\delta f$ in terms of the moment variables, the definitions of the latter (hence which moments to take), and the closure rules are already defined. We simply take the moment list defined in Eqs. (43) and apply each one separately to Eq. (28). With one exception, all the steps follow directly. The exception is the parallel magnetic nonlinearities, in which both field variables appear, each with a factor of $J_{0}$, and the velocity space integral must be decided. But as we will see, the form this must have is already determined by the requirement of energy conservation.

\section{A. Toroidal curvature, magnetic divergence effects}

The delta-f form of the gyrokinetic equation splits the curvature and grad-B drifts from the rest, as these are purely linear terms. These two drifts are combined, such that in each case we take a combined moment over $m z^{2}+w B$. The quantity operated upon is $h$, not $\delta f$, so that the field potential moments also appear. Only $\phi$ is involved, and $J_{0}$ appears only with $\phi$. Moreover, only derivatives over $x$ and $y$ are involved, while $B$ depends only on $s$. These terms are therefore found by simple evaluation of the moments over $m z^{2}+w B$ times $h$. These terms conserve energy separately from those arising from other brackets, as in the delta-f Vlasov equation (in Eq. 28 combines with itself under $\mathcal{K}$ as a pure divergence). The 4th moment $\left(w^{2} B^{2}\right)$ over $J_{0} \phi$ in Eq. (62) is determined by this requirement, as noted there. After evaluation of the moments, the curvature terms appear as

$$
\begin{aligned}
& \frac{\partial}{\partial t} \frac{\tilde{n}}{n}=\cdots+\frac{T}{e} \mathcal{K}\left(\frac{\widetilde{p}_{\|}+\widetilde{p}_{\perp}}{2 n T}+\frac{e \phi_{G}}{T}+\frac{e \Omega_{G}}{2 T}\right) \\
& \frac{\partial}{\partial t}\left(\frac{1}{c} A_{G}+\frac{m}{e} \widetilde{u}_{\|}\right)=\cdots+\frac{m T}{e^{2}} \mathcal{K}\left(2 \widetilde{u}_{\|}+\frac{2 \widetilde{q}_{\|}+\widetilde{q}_{\perp}}{2 n T}\right)
\end{aligned}
$$




$$
\begin{aligned}
& \frac{1}{2} \frac{\partial}{\partial t} \frac{\widetilde{T}_{\|}}{T}=\cdots+\frac{T}{e} \mathcal{K}\left(\frac{\widetilde{p}_{\|}}{2 n T}+\frac{e \phi_{G}}{2 T}+\frac{\widetilde{T}_{\|}}{T}\right) \\
& \frac{\partial}{\partial t} \frac{\widetilde{T}_{\perp}}{T}=\cdots+\frac{T}{e} \mathcal{K}\left(\frac{\widetilde{p}_{\perp}}{2 n T}+\frac{e \phi_{G}+e \Omega_{G}}{2 T}+3 \frac{\widetilde{T}_{\perp}+e \Omega_{G}}{2 T}\right) \\
& \frac{\partial}{\partial t}\left(\frac{m}{e} \frac{\widetilde{q}_{\|}}{n T}\right)=\cdots+\frac{m T}{e^{2}} \mathcal{K}\left(\frac{3}{2} \widetilde{u}_{\|}+4 \frac{\widetilde{q}_{\|}}{n T}\right) \\
& \frac{\partial}{\partial t}\left(\frac{1}{c} \chi_{G}+\frac{m}{e} \frac{\widetilde{q}_{\perp}}{n T}\right)=\cdots+\frac{m T}{e^{2}} \mathcal{K}\left(\frac{1}{2} \widetilde{u}_{\|}+3 \frac{\widetilde{q}_{\perp}}{n T}\right)
\end{aligned}
$$

The terms with $\mathcal{K}$ operating directly on the moment variables always appear with factors of $T / e$ in these scaled units, reflecting the charge separation effect of "diamagnetic" curvature terms. Those acting on $\phi$ give the ExB compression effects plus FLR corrections. In the parallel flux variable equations there is the additional factor of $m / e$ matching the one in the inertia terms. These curvature terms form a closed set which conserves energy separately, as detailed in Ref. [15].

\section{B. Nonlinear ExB advection}

In the nonlinear brackets $\left[\left(J_{0} \phi\right),(\delta f)\right]_{x y}$ both $\phi$ and $\delta f$ appear under gradients. The moment mixing for state variables is given by

$$
\begin{gathered}
\int d \mathcal{W}\left[\left(J_{0} \phi\right),(\delta f)\right]_{x y}=\left[\phi_{G}, \widetilde{n}_{x y}+\frac{n}{T}\left[\Omega_{G}, \widetilde{T}_{\perp}\right]_{x y}\right. \\
\int d \mathcal{W}(w B-T)\left[\left(J_{0} \phi\right),(\delta f)\right]_{x y}=n\left[\phi_{G}, \widetilde{T}_{\perp}\right]_{x y}+\left[\Omega_{G},\left(T \widetilde{n}+2 n \widetilde{T}_{\perp}\right)\right]_{x y}
\end{gathered}
$$

For flux variables it is similar,

$$
\begin{gathered}
\int d \mathcal{W} z\left[\left(J_{0} \phi\right),(\delta f)\right]_{x y}=n\left[\phi_{G}, \widetilde{u}_{\|}\right]_{x y}+\left[\Omega_{G}, \frac{\widetilde{q}_{\perp}}{T}\right]_{x y} \\
\int d \mathcal{W} z(w B-T)\left[\left(J_{0} \phi\right),(\delta f)\right]_{x y}=\left[\phi_{G}, \widetilde{q}_{\perp}\right]_{x y}+\left[\Omega_{G},\left(n T \widetilde{u}_{\|}+2 \widetilde{q}_{\perp}\right)\right]_{x y}
\end{gathered}
$$

For the pure parallel velocity moments there is no mixing as no factors of $w B$ appear,

$$
\begin{gathered}
\int d \mathcal{W}\left(m z^{2}-T\right)\left[\left(J_{0} \phi\right),(\delta f)\right]_{x y}=n\left[\phi_{G}, \widetilde{T}_{\|}\right]_{x y} \\
\int d \mathcal{W} \frac{z}{2}\left(m z^{2}-T\right)\left[\left(J_{0} \phi\right),(\delta f)\right]_{x y}=\left[\phi_{G}, \widetilde{q}_{\|}\right]_{x y}
\end{gathered}
$$

The nonlinearities involving $A_{\|}$properly belong to the parallel dynamics. Since the parallel gradient ultimately acts on $h$ rather than $\delta f$ in the kinetic model, these nonlinearities involve 
two appearances of the field potentials $\left(A_{\|}\right.$and $\phi$, in the nonlinear parallel electric field). In the ExB advection terms these pieces vanish because they all arise from $\left[\left(J_{0} \phi\right),\left(J_{0} \phi\right)\right]_{x y}$. Indeed, if we apply the rules for $h$ to the above six combinations $\left(\phi_{G}\right.$ with $\widetilde{n}$ and $\Omega_{G}$ with $\widetilde{T}_{\perp}$, as in Eq. 56$)$, all the terms quadratic in $\phi$ vanish, as they should do. But for $\left[\left(J_{0} A_{\|}\right),\left(J_{0} \phi\right)\right]_{x y}$ several field terms survive (the lowest order among them being the magnetic flutter effect on the electric field in the Ohm's law, in the fluid sense) and the energy theorem's constraints are needed to evaluate them. They are left to the discussion on nonlinear parallel dynamics, which follows next.

\section{Parallel dynamics and magnetic nonlinearities}

In the gyrokinetic nonlinear bracket both $\phi$ and $A_{\|}$appear in the gyrokinetic potential (perturbed Hamiltonian). Since $A_{\|}$appears with an extra factor of $z$, we compute these terms separately as they involve different moments. The lowest order $A_{\|}$terms combine in the nonlinear parallel gradient,

$$
\nabla_{\|}=\frac{B^{s}}{B} \frac{\partial}{\partial s}-\left[A_{G},\right]_{x y}
$$

to which the $\Gamma_{2}$-dependent FLR effects add and mix moments in the same way as with $\phi_{G}$ and $\Omega_{G}$ in the ExB advection. However, the $\partial / \partial s$ terms also involve the dependence $B=B(s)$, so it is useful to consider these separately.

\section{Linear parallel dynamics}

The linear terms arise from the $\left[H_{0}, h\right]_{z s}$ bracket in Eq. (28), whose two pieces combine parallel streaming and magnetic trapping effects. Here, $B$ does not commute with $\partial / \partial s$ so there arise extra terms proportional to $\partial B / \partial s$, referred to as magnetic pumping terms, the vestige of kinetic trapping in the gyrofluid model [1]. There is also the distinction between a parallel divergence and a parallel gradient, according to whether $1 / B$ occurs inside or outside of $\partial / \partial s$. As with the curvature terms, the moments are over $h$, not $\delta f$, so the same combinations arise as in the curvature terms. With these extra considerations the

moment calculations are straightforward. Again, these terms conserve energy separately and hence can be considered separately (with the magnetic pumping terms independent of 
the others). After evaluation of the moments, the linear parallel gradient/divergence and magnetic pumping terms appear as

$$
\begin{aligned}
& \frac{\partial}{\partial t} \frac{\widetilde{n}}{n}=\cdots-B \nabla_{\|}^{0} \frac{\widetilde{u}_{\|}}{B} \\
& \frac{\partial}{\partial t}\left(\frac{1}{c} A_{G}+\frac{m}{e} \widetilde{u}_{\|}\right)=\cdots-\nabla_{\|}^{0} \frac{n e \phi_{G}+T \widetilde{n}+n \widetilde{T}_{\|}}{n e}-\frac{\left(e \Omega_{G}+\widetilde{T}_{\perp}\right)-\widetilde{T}_{\|}}{e} \nabla_{\|}^{0} \log B \\
& \frac{1}{2} \frac{\partial}{\partial t} \frac{\widetilde{T}_{\|}}{T}=\cdots-B \nabla_{\|}^{0} \frac{n T \widetilde{u}_{\|}+\widetilde{q}_{\|}}{n T B}-\frac{n T \widetilde{u}_{\|}+\widetilde{q}_{\perp}}{n T} \nabla_{\|}^{0} \log B \\
& \frac{\partial}{\partial t} \frac{\widetilde{T}_{\perp}}{T}=\cdots-B \nabla_{\|}^{0} \frac{\widetilde{q}_{\perp}}{n T B}+\frac{n T \widetilde{u}_{\|}+\widetilde{q}_{\perp}}{n T} \nabla_{\|}^{0} \log B \\
& \frac{\partial}{\partial t}\left(\frac{m}{e} \frac{\widetilde{q}_{\|}}{n T}\right)=\cdots-\frac{3}{2} \nabla_{\|}^{0} \frac{\widetilde{T}_{\|}}{e} \\
& \frac{\partial}{\partial t}\left(\frac{1}{c} \chi_{G}+\frac{m}{e} \frac{\widetilde{q}_{\perp}}{n T}\right)=\cdots-\nabla_{\|}^{0} \frac{e \Omega_{G}+\widetilde{T}_{\perp}}{e}-\frac{\left(e \Omega_{G}+\widetilde{T}_{\perp}\right)-\widetilde{T}_{\|}}{e} \nabla_{\|}^{0} \log B
\end{aligned}
$$

where $\nabla_{\|}^{0}$ denotes the linear part of the parallel gradient. In field-aligned Hamada coordinates it is given by

$$
B \nabla_{\|}^{0}=B^{s} \frac{\partial}{\partial s}
$$

with $B^{s}$ independent of $s$. The Jacobian does not appear because it is a flux function (function of $x$ only). Under strict delta-f ordering both the Jacobian and $B^{s}$ are constants. In general the Jacobian enters, so that

$$
\nabla_{\|}^{0}=\frac{B^{s}}{B} \frac{\partial}{\partial s} \quad B \nabla_{\|}^{0} \frac{f}{B}=\frac{1}{\sqrt{g}} \frac{\partial}{\partial s}\left(\sqrt{g} \frac{B^{s}}{B} f\right)
$$

where $g$ is the determinant of the metric coefficients (all three components). It is essential that $\sqrt{g} B^{s}$ be a flux function, to preserve $\nabla \cdot \mathbf{B}=0$.

\section{E. Nonlinear parallel dynamics}

The lowest-order nonlinear terms follow by consistency from the linear ones, as both pieces of the nonlinear parallel derivative $\nabla_{\|}=\nabla_{\|}^{0}-\left[A_{G},\right]_{x y}$ act together. However, treating temperature dynamics with FLR consistency adds FLR nonlinearities to these, involving brackets with $\chi_{G}$. These involve moment mixing in the same way as for $\phi_{G}$ and $\Omega_{G}$ in the ExB advection. The moment integrals encountered are basically the same, because the integrals over $z$ and $w$ separate, and the parallel dynamics is merely one order higher by the factor of $z$ in the moment hierarchy. 
Terms arising from $\left[\left(J_{0} A_{\|}\right),(\delta f)\right]_{x y}$ involving $A_{G}$ simply follow from the linear ones arising from $\nabla_{\|}^{0}(\delta f)$. Those involving $\chi_{G}$ raise the moment level by one in $w$ in the same way as in the ExB advection terms. The only subtlety is the one involving moments over the field nonlinearity bracket $\left[J_{0} A_{\|}, J_{0} \phi\right]_{x y}$ which is unique to the nonlinear parallel dynamics (the corresponding terms in ExB advection vanish trivially with $\left[J_{0} \phi, J_{0} \phi\right]_{x y}$ ). These terms appear in the flux variable equations,

$$
\begin{aligned}
& \frac{\partial}{\partial t}\left(\frac{1}{c} A_{G}+\frac{m}{e} \widetilde{u}_{\|}\right)=\cdots+\int d \mathcal{W} m z \frac{F^{M}}{n T} \frac{z}{B_{0}}\left[J_{0} A_{\|}, J_{0} \phi\right]_{x y} \\
& \frac{\partial}{\partial t}\left(\frac{m}{e} \frac{\widetilde{q}_{\|}}{n T}\right)=\cdots+\int d \mathcal{W} \frac{m z^{2}-T}{T} \frac{m z}{2} \frac{F^{M}}{n T} \frac{z}{B_{0}}\left[J_{0} A_{\|}, J_{0} \phi\right]_{x y} \\
& \frac{\partial}{\partial t}\left(\frac{1}{c} \chi_{G}+\frac{m}{e} \frac{\widetilde{q}_{\perp}}{n T}\right)=\cdots+\int d \mathcal{W} \frac{w B-T}{T} m z \frac{F^{M}}{n T} \frac{z}{B_{0}}\left[J_{0} A_{\|}, J_{0} \phi\right]_{x y}
\end{aligned}
$$

since in the state variable equations they vanish due to the odd symmetry of $\int d \mathcal{W} z$. The difficulty is that the velocity space integration is no longer associated with only one of the quantities appearing under spatial derivatives in the bracket. The solution is to apply the moments to the state variable terms in the $(\delta f)$ representation in Eq. (44) first. These give

$$
\begin{aligned}
& \frac{\partial}{\partial t}\left(\frac{1}{c} A_{G}+\frac{m}{e} \widetilde{u}_{\|}\right)=\cdots+\frac{1}{n e B_{0}}\left[A_{G}, \widetilde{p}_{\|}\right]_{x y}+\frac{1}{e B_{0}}\left[\chi_{G}, \widetilde{T}_{\perp}\right]_{x y} \\
& \frac{\partial}{\partial t}\left(\frac{m}{e} \frac{\widetilde{q}_{\|}}{n T}\right)=\cdots+\frac{3}{2 e B_{0}}\left[A_{G}, \widetilde{T}_{\|}\right]_{x y} \\
& \frac{\partial}{\partial t}\left(\frac{1}{c} \chi_{G}+\frac{m}{e} \frac{\widetilde{q}_{\perp}}{n T}\right)=\cdots+\frac{1}{e B_{0}}\left[A_{G}, \widetilde{T}_{\perp}\right]_{x y}+\frac{1}{n e B_{0}}\left[\chi_{G},\left(\widetilde{p}_{\|}+2 n \widetilde{T}_{\perp}\right)\right]_{x y}
\end{aligned}
$$

Now we apply the combination rules for $h$ given in Eq. (56), so that these become

$$
\begin{aligned}
\frac{\partial}{\partial t}\left(\frac{1}{c} A_{G}\right. & \left.+\frac{m}{e} \widetilde{u}_{\|}\right)=\cdots+\frac{1}{n e B_{0}}\left[A_{G},\left(\widetilde{p}_{\|}+n e \phi_{G}\right)\right]_{x y} \\
& +\frac{1}{e B_{0}}\left[\chi_{G},\left(\widetilde{T}_{\perp}+e \Omega_{G}\right)\right]_{x y} \\
\frac{\partial}{\partial t}\left(\frac{m}{e} \frac{\widetilde{q}_{\|}}{n T}\right) & =\cdots+\frac{3}{2 e B_{0}}\left[A_{G}, \widetilde{T}_{\|}\right]_{x y} \\
\frac{\partial}{\partial t}\left(\frac{1}{c} \chi_{G}\right. & \left.+\frac{m}{e} \frac{\widetilde{q}_{\perp}}{n T}\right)=\cdots+\frac{1}{e B_{0}}\left[A_{G},\left(\widetilde{T}_{\perp}+e \Omega_{G}\right)\right]_{x y} \\
& +\frac{1}{n e B_{0}}\left[\chi_{G},\left(\widetilde{p}_{\|}+n e \phi_{G}\right)\right]_{x y}+\frac{2}{e B_{0}}\left[\chi_{G},\left(\widetilde{T}_{\perp}+e \Omega_{G}\right)\right]_{x y}
\end{aligned}
$$

Hence we have determined that the rules for moments over two operations by $J_{0}$ on field variables are

$$
\frac{1}{n} \int d \mathcal{W} F^{M}\left[\left(J_{0} A_{\|}\right),\left(J_{0} \phi\right)\right]=\left[\left(\Gamma_{1} A_{\|}\right),\left(\Gamma_{1} \phi\right)\right]+\left[\left(\Gamma_{2} A_{\|}\right),\left(\Gamma_{2} \phi\right)\right]
$$




$$
\left.\frac{1}{n} \int d \mathcal{W} \frac{w B}{T} F^{M}\left[\left(J_{0} A_{\|}\right),\left(J_{0} \phi\right)\right]=\left[\left(\Gamma_{1}+\Gamma_{2}\right) A_{\|}, \Gamma_{2} \phi\right)\right]+\left[\Gamma_{2} A_{\|},\left(\Gamma_{1}+\Gamma_{2}\right) \phi\right]
$$

It is simple to show that these vanish if $A_{\|} \rightarrow \phi$, as they should do.

In the state variable equations the $A_{\|}, \phi$ combinations do not appear, and the nonlinear parallel divergences of flux variables are left as

$$
\begin{aligned}
& \frac{\partial}{\partial t} \frac{\widetilde{n}}{n}=\cdots+\frac{1}{B_{0}}\left[A_{G}, \widetilde{u}_{\|}\right]_{x y}+\frac{1}{n T B_{0}}\left[\chi_{G}, \widetilde{q}_{\perp}\right]_{x y} \\
& \frac{1}{2} \frac{\partial}{\partial t} \frac{\widetilde{T}_{\|}}{T}=\cdots+\frac{1}{n T B_{0}}\left[A_{G},\left(n T \widetilde{u}_{\|}+\widetilde{q}_{\|}\right)\right]_{x y}+\frac{1}{n T B_{0}}\left[\chi_{G}, \widetilde{q}_{\perp}\right]_{x y} \\
& \frac{\partial}{\partial t} \frac{\widetilde{T}_{\perp}}{T}=\cdots+\frac{1}{n T B_{0}}\left[A_{G}, \widetilde{q}_{\perp}\right]_{x y}+\frac{1}{n T B_{0}}\left[\chi_{G},\left(n T \widetilde{u}_{\|}+2 \widetilde{q}_{\perp}\right)\right]_{x y}
\end{aligned}
$$

Here, there is crosstalk between $\widetilde{n}$ or $\widetilde{T}_{\|}$and $\widetilde{q}_{\perp}$ due to the fact that the corresponding $w B-T$ moment does not vanish when a factor of $J_{0}$ is present. Again, this set of terms conserves energy separately from all the others. By contrast to ExB advection, however, the conservation is between state and flux variable sets, not just within each of those sets. When the flux variables are kept as time dependent variables, the level of moment mixing within state and flux variable sets remains consistent (hence velocity with density and heat flux with temperature, with one heat flux for each of perpendicular and parallel temperatures). With these terms determined, the dissipation free part of the equations, the part which conserves free energy exactly, is now closed. Under the strict delta-f ordering, with the geometry dependent upon $s$ and with nonlinear derivatives only in $\{x, y\}$, the constraint $\nabla \cdot \mathbf{B}=0$ is maintained in the nonlinear terms as well.

\section{DISSIPATIVE EFFECTS}

The addition of dissipation is essentially done by hand, as in most of the moment approaches (cf. Ref. [1]), as it is not part of the original Lagrangian/Hamiltonian formulation. Even at the gyrokinetic level, if collisional effects are very weak then either the computation must resolve very thin striations in phase space (cf. Refs. [38 41]), or it must cut them off via a hyperdiffusion in velocity space which accounts for what is essentially the same thing as a "high Reynolds number" situation viz-a-viz Fokker-Planck collisional diffusion [42, 43]. Here we note that the basic mathematical properties of the one-dimensional (1D) Vlasov-Maxwell system of the earlier references are the same as the shear-Alfvén subset 
of the electromagnetic gyrokinetic turbulence discussed in the more recent ones. The general gyrokinetic collisional process is being developed on more firm mathematical grounds [44, 45], but it is mostly simplified models that are currently in use [46], when collisions are used at all.

Dissipation effects due to both Landau damping and collisions are added to the gyrofluid equations at more or less the same level of sophistication. While it is possible to mimic the linear response of the plasma dispersion function in the linear limit in homogeneous geometry [2, 3] or for simplified instabilities in toroidal geometry [1], a general fitting approach was shown to fail even for linear instabilities close to and away from threshold, by the necessity to use a different fitting matrix for each case [1]. Hence, Landau damping was inserted into the heat flux equations as a direct-damping model in Ref. [15], such that the Alfvén damping response is adequately captured without impacting any of the conservative transfer processes (i.e., by changing their coefficients, as was done in Ref. [2]).

Collisions are another matter, as there is a well formulated fluid limit to which the equations should relax if the collisional frequency becomes large compared to any parallel transit or nonlinear advection frequencies — the Braginskii equations [10]. Typical fluid models in the drift frequency regime keep the parallel dissipation effects (resistivity, thermal forces, parallel viscosity) [47-49]. Gyrofluid equations, functioning in the same regime, keep these same processes as well [4, 15]. The only complication is to keep them consistent with the anisotropic temperature model, and to formulate the thermal forces such that they disappear naturally when the collision frequency drops to zero [47]. At the drift kinetic or gyrokinetic level, the dissipation is covered by the 1-D part of the equations describing parallel dynamics, and a simple procedure has been shown for the drift kinetic equation for electrons in Ref. [50] which we can use here. This is to set up a Chapman-Enskog expansion around a simple Lorentz collision operator, obtain directly all the necessary terms, and then change the coefficients such that the result agrees with the Braginskii model. Here, the only extension of that was to start from a bi-Maxwellian with given perpendicular and parallel temperatures

$$
F^{(0)}=n\left(2 \pi T_{\|} / m\right)^{-1 / 2}\left(2 \pi T_{\perp} / m\right)^{-1} \exp \left(-w B / T_{\perp}-m z^{2} / 2 T_{\|}\right)
$$

and then to solve the correction equation

$$
\left(z \nabla_{\|}+\frac{e}{m} E_{\|} \frac{\partial}{\partial z}\right) F^{(0)}=\frac{\nu_{L}}{v^{3}} \frac{\partial}{\partial \zeta}\left(1-\zeta^{2}\right) \frac{\partial f^{(1)}}{\partial \zeta}
$$


for $f^{(1)}$, where coordinates $\{v, \zeta\}$ are given by $m v^{2}=2 w B+m z^{2}$ and $\zeta=z / v$, and $\nu_{L}$ is the Lorentz collision parameter. Following Ref. [50], at lowest order there are no flows (in the local rest frame of the ion fluid), so the consistency conditions by which $\partial / \partial t$ is eliminated are trivial (small parallel force imbalance, no divergences). Also, a finite $A_{\|}$is neglected by assuming the resistivity is sufficiently large, and toroidal drifts are neglected by assuming $R \gg L_{\perp}$, where $L_{\perp}$ is the profile scale length. Having solved for $f^{(1)}$, the heat flux variables $\widetilde{q}_{\|\|}$and $\widetilde{q}_{\perp}$ and, for electrons, the parallel current $J_{\|}=-n_{e} e u_{e \|}$ are evaluated directly. This yields collisional formulae for the flux variables

$$
\begin{aligned}
& \eta \frac{m_{e} \nu_{e}}{e} J_{\|}=n_{e} e E_{\|}+\nabla_{\|} p_{e \|}+\alpha_{e} n_{e} \nabla_{\|} T_{e \|} \\
& q_{e\|\|}+1.28\left(q_{e\|\|}-1.5 q_{e \perp \|}\right)+\frac{3}{5} \alpha_{e} \frac{T_{e}}{e} J_{\|}=-\frac{3}{5} \kappa_{e} \frac{T_{e}}{m_{e} \nu_{e}} n_{e} \nabla_{\|} T_{e \|} \\
& q_{e \perp \|}-1.28\left(q_{e\|\|}-1.5 q_{e \perp \|}\right)+\frac{2}{5} \alpha_{e} \frac{T_{e}}{e} J_{\|}=-\frac{2}{5} \kappa_{e} \frac{T_{e}}{m_{e} \nu_{e}} n_{e} \nabla_{\|} T_{e \perp}
\end{aligned}
$$

as well as anisotropy dissipation corrections to the temperature equations

$$
\begin{aligned}
& \frac{1}{2} n_{e} \frac{\partial T_{e \|}}{\partial t}+\cdots=-\frac{\nu_{e}}{3 \pi_{e}}\left(T_{e \|}-T_{e \perp}\right) \\
& n_{e} \frac{\partial T_{e \perp}}{\partial t}+\cdots=\frac{\nu_{e}}{3 \pi_{e}}\left(T_{e \|}-T_{e \perp}\right)
\end{aligned}
$$

Here, the numerical coefficients resulting from the Lorentz model which are kept are written explicitly, while the coefficients to be substituted with their Braginskii values are written as $\eta$, $\alpha_{e}, \kappa_{e}$, and $\pi_{e}$, for resistivity, thermoelectric coupling, thermal conduction, and (eventually) parallel viscosity, respectively. As the subscripts indicate, the calculation itself is done for electrons. For ions, the thermoelectric coupling is set to zero, and then $\kappa_{i}$ and $\pi_{i}$ are given their Braginskii values (for several ion species if desired).

To treat the transcollisional situation the thermoelectric coupling is reformulated (obviously, it should vanish as $\nu_{e} \rightarrow 0$ ). The thermal force itself, $\alpha_{e} n_{e} \nabla_{\|} T_{e \|}$, is substituted using the heat flux formulae (in this, the anisotropy is assumed to be small for large $\nu_{e}$ ), as detailed in Ref. [47], so that the electron force imbalance is entirely due to dissipation of the flux variables,

$$
n_{e} e\left(\frac{1}{c} \frac{\partial A_{\|}}{\partial t}+\nabla_{\|} \phi\right)-\nabla_{\|} p_{e \|}=-m_{e} \nu_{e}\left[\eta \frac{J_{\|}}{e}+\frac{\alpha_{e}}{\kappa_{e}}\left(\frac{q_{e}\|\|+q_{e \perp \|}}{T_{e}}+\alpha_{e} \frac{J_{\|}}{e}\right)\right]
$$


having The heat flux formulae are treated in a similar fashion,

$$
\begin{aligned}
& \frac{3}{2} p_{e} \nabla_{\|} T_{e \|}=-m_{e} \nu_{e} \frac{5 / 2}{\kappa_{e}}\left[q_{e\|\|}+1.28\left(q_{e\|\|}-1.5 q_{e \perp \|}\right)+\frac{3}{5} \alpha_{e} \frac{T_{e}}{e} J_{\|}\right] \\
& p_{e} \nabla_{\|} T_{e \perp}=-m_{e} \nu_{e} \frac{5 / 2}{\kappa_{e}}\left[q_{e \perp \|}-1.28\left(q_{e\|\|}-1.5 q_{e \perp \|}\right)+\frac{2}{5} \alpha_{e} \frac{T_{e}}{e} J_{\|}\right]
\end{aligned}
$$

with gradients on the left and dissipative damping terms on the right. The right hand sides of Eqs. (117 121) represent a set of terms which are added to the right hand sides to the corresponding gyrofluid moment equations. The resistivity combination in Eq. (119) subtracts from the right hand side of $\partial A_{G} / \partial t$. The temperature terms in Eqs. (117]118) add to the temperature equations as shown. The heat flux terms in Eqs. (120,121) add to the equations for $q_{e\|\|}$ and $q_{e \perp \|}$, respectively. The ions are done the same way as the electrons except for the resistivity terms (which are the same for all species), and with the coefficients $\kappa_{i}$ and $\pi_{i}$ the appropriate ones for each ion species, with $\alpha$ set to zero for the ions.

Parallel viscosity does not explicitly appear in the equation for the parallel velocity moment but instead results from collisional dissipation of the difference between perpendicular and parallel temperatures. This difference is of course one and the same with the parallelparallel component of the viscous tensor [10]. Either one keeps a viscosity term in the parallel momentum equation or one keeps track of thermal anisotropy with the dependent variables, but not both. There is some discussion of this in Refs. [1, 2], and it is the ultimate reason that no Landau closure dissipative term should appear in the parallel velocity equation itself [3]. Such a two moment dissipative closure has been given [5], but especially for electrons it has the undesirable property of mimicking a resistivity which is a factor of $V_{e} / \nu_{e} q R$ too large. Hence the Landau closure itself appears only in the heat flux moment equations, following Refs. [1, 2, 15], and the parallel viscosity is naturally given by the collisional dissipation of thermal anisotropy if the collision frequency is sufficiently dominant [51]. The Chapman-Enskog procedure gives the coefficient $\nu_{z} / 3$ for species $z$, as in Ref. [1]. The coefficient $\pi_{z}$ then gives the correct viscosity coefficient for species $z$ according to the collisional fluid derivation [10].

The correspondence of these equations to low frequency fluid drift equations including nonlinear polarisation and collisional dissipation processes in the appropriate ("Braginskii") regime has been shown elsewhere and a summary is given in Sec. VIIID, below. 


\section{RESULTING GYROFLUID EQUATIONS}

The results of the above derivation are essentially the same as those of Ref. [15]. The two new elements are the derivation path and the inclusion of direct finite gyroradius effects in the part of the nonlinear dynamics representing the fluctuations in the magnetic field. The derivation is now firmly grounded within the underlying gyrokinetic theory to the extent that the representation of the gyrokinetic distribution function in terms of the gyrofluid moment variables is explicit and the energy theorem not only remains intact but has itself been used to determine the closure rules. The part of the gyrofluid moment equations which

arises directly from the gyrokinetic model and is exactly conservative is given by equations for the gyrocenter density,

$$
\frac{d n_{z}}{d t}+\left[\Omega_{G}, T_{z \perp}\right]+B \nabla_{\|} \frac{u_{z \|}}{B}-\beta_{e}\left[\chi_{G}, q_{z \perp}\right]=\mathcal{K}\left(\tau_{z} \frac{p_{z \|}+p_{z \perp}}{2}+\phi_{G}+\frac{\Omega_{G}}{2}\right)
$$

parallel velocity,

$$
\begin{gathered}
\beta_{e} \frac{\partial A_{G}}{\partial t}+\mu_{z} \frac{d u_{z \|}}{d t}+\mu_{z}\left[\Omega_{G}, q_{z \perp}\right]+\nabla_{\|}\left(\phi_{G}+\tau_{z} p_{z \|}\right)-\beta_{e}\left[\chi_{G},\left(\Omega_{G}+\tau_{z} T_{z \perp}\right)\right] \\
+\left(\Omega_{G}+\tau_{z} T_{z \perp}-\tau_{z} T_{z \|}\right) \nabla_{\|} \log B=\tau_{z} \mu_{z} \mathcal{K}\left(\frac{4 u_{z \|}+2 q_{z \|}+q_{z \perp}}{2}\right)
\end{gathered}
$$

parallel temperature,

$$
\begin{aligned}
\frac{1}{2} \frac{d T_{z \|}}{d t} & +B \nabla_{\|} \frac{u_{z \|}+q_{z \|}}{B}-\beta_{e}\left[\chi_{G}, q_{z \perp}\right]+\left(u_{z \|}+q_{z \perp}\right) \nabla_{\|} \log B \\
= & \mathcal{K}\left(\tau_{z} \frac{p_{z \|}+2 T_{z \|}}{2}+\frac{\phi_{G}}{2}\right)
\end{aligned}
$$

perpendicular temperature,

$$
\begin{aligned}
\frac{d T_{z \perp}}{d t} & +\left[\Omega_{G},\left(n_{z}+2 T_{z \perp}\right)\right] \\
& +B \nabla_{\|} \frac{q_{z \perp}}{B}-\beta_{e}\left[\chi_{G},\left(u_{z \|}+2 q_{z \perp}\right)\right]-\left(u_{z \|}+q_{z \perp}\right) \nabla_{\|} \log B \\
& =\mathcal{K}\left(\tau_{z} \frac{p_{z \perp}+3 T_{z \perp}}{2}+\frac{\phi_{G}+4 \Omega_{G}}{2}\right)
\end{aligned}
$$

parallel/parallel heat flux,

$$
\mu_{z} \frac{d q_{z \|}}{d t}+\frac{3}{2} \tau_{z} \nabla_{\|} T_{z \|}=\tau_{z} \mu_{z} \mathcal{K}\left(\frac{3 u_{z \|}+8 q_{z \|}}{2}\right)
$$

and perp/parallel heat flux,

$$
\beta_{e} \frac{\partial \chi_{G}}{\partial t}+\mu_{z} \frac{d q_{z \perp}}{d t}+\mu_{z}\left[\Omega_{G},\left(u_{z \|}+2 q_{z \perp}\right)\right]
$$




$$
\begin{aligned}
& +\tau_{z} \nabla_{\|} T_{z \perp}-\beta_{e}\left[\chi_{G},\left(\phi_{G}+\tau_{z} p_{z \|}\right)\right]-\beta_{e}\left[\chi_{G}, 2\left(\Omega_{G}+\tau_{z} T_{z \perp}\right)\right] \\
& +\left(\Omega_{G}+\tau_{z} T_{z \perp}-\tau_{z} T_{z \|}\right) \nabla_{\|} \log B=\tau_{z} \mu_{z} \mathcal{K}\left(\frac{u_{z \|}+6 q_{z \perp}}{2}\right)
\end{aligned}
$$

The lowest order nonlinear advective and parallel derivatives are given by

$$
\frac{d}{d t}=\frac{\partial}{\partial t}+\left[\phi_{G},\right] \quad \nabla_{\|}=\mathbf{b} \cdot \nabla-\beta_{e}\left[A_{G},\right]
$$

with $\mathbf{b}$ the unit vector of the unperturbed magnetic field.

The nonlinear brackets are given by

$$
[f, g]=\nabla f \cdot \mathbf{F}_{0} \cdot \nabla g \quad \text { given } \quad \nabla \cdot \mathbf{F}_{0}=0 \quad \text { and } \quad \mathbf{F}_{0}: \nabla \nabla=0
$$

where $\mathbf{F}_{0}$ is a divergence free, antisymmetric tensor as specified, in the particular normalisation being used. Similarly for the curvature operator,

$$
\mathcal{K}(f)=\mathcal{K}^{i} \nabla_{i} f \quad \text { given } \quad \nabla_{i} \mathcal{K}^{i}=0
$$

A typical case is to leave the drift scale ratio $\rho_{s} / L_{\perp}$ out of the normalisation and put it into $\mathbf{F}_{0}$ and $\mathcal{K}$. Conventional normalisation is to fold it into the normalisation, keep to strict fluxtube ordering, so that $[f, g]=f_{, x} g_{, y}-f_{, y} g_{, x}$ in the conventional linearised gyro-Bohm version as in Refs. [1, 2]. Both versions are covered in Ref. [15].

The FLR reduced potentials are given by

$$
\begin{array}{ll}
\phi_{G}=\Gamma_{1} \phi & A_{G}=\Gamma_{1} A_{\|} \\
\Omega_{G}=\Gamma_{2} \phi & \chi_{G}=\Gamma_{2} A_{\|}
\end{array}
$$

in terms of the field potentials $\phi$ and $A_{\|}$. The associated field potential equations are given by

$$
\sum_{z} a_{z}\left[\Gamma_{1} n_{z}+\Gamma_{2} T_{z \perp}+\frac{\Gamma_{0}-1}{\tau_{z}} \phi\right]=0
$$

for polarisation and

$$
\nabla_{\perp}^{2} A_{\|}+\sum_{z} a_{z}\left[\Gamma_{1} u_{z \|}+\Gamma_{2} q_{z \perp}\right]=0
$$

for induction. In a computational model the induction equation is actually solved using the combinations under the $\partial / \partial t$ in Eqs. (123], 127)

$$
\begin{aligned}
\left(\sum_{z} a_{z}\left[\frac{\beta_{e}}{\mu_{z}}\left(\Gamma_{1}^{2}+\Gamma_{2}^{2}\right)\right]-\nabla_{\perp}^{2}\right) A_{\|} \\
\quad=\sum_{z} \frac{a_{z}}{\mu_{z}}\left[\Gamma_{1}\left(\beta_{e} A_{G}+\mu_{z} u_{z \|}\right)+\Gamma_{2}\left(\beta_{e} \chi_{G}+\mu_{z} q_{z \perp}\right)\right]
\end{aligned}
$$


as these quantities on the right hand side are what are actually advanced.

The constant parameters

$$
a_{z}=\frac{n_{z} Z}{n_{e}} \quad \tau_{z}=\frac{T_{z}}{Z T_{e}} \quad \mu_{z}=\frac{m_{z}}{Z m_{D}}
$$

given the background charge density, temperature/charge, and mass/charge ratios, normalised to electron and deuterium values. The pressures are linearised, so that

$$
p_{z \|}=n_{z}+T_{z \|} \quad p_{z \perp}=n_{z}+T_{z \perp}
$$

under gradient operators. The profile gradients for all the state variables are included as part of the dependent variables; these may be split in a traditional manner without loss of generality.

To these equations are added the collisional dissipation model,

$$
\begin{aligned}
& \beta_{e} \frac{\partial A_{G}}{\partial t}+\mu_{z} \frac{\partial u_{z \|}}{\partial t}=\cdots-\mu_{e} \nu_{e}\left[\eta J_{\|}+\frac{\alpha_{e}}{\kappa_{e}}\left(q_{e \|}+q_{e \perp}+\alpha_{e} J_{\|}\right)\right] \\
& \frac{1}{2} \frac{\partial T_{z \|}}{\partial t}=\cdots-\frac{\nu_{z}}{3 \pi_{z}}\left(T_{z \|}-T_{z \perp}\right) \\
& \frac{\partial T_{z \perp}}{\partial t}=\cdots+\frac{\nu_{z}}{3 \pi_{z}}\left(T_{z \|}-T_{z \perp}\right) \\
& \mu_{z} \frac{\partial q_{z \|}}{\partial t}=\cdots-\mu_{z} \nu_{z} \frac{5 / 2}{\kappa_{e}}\left[q_{z \|}+1.28\left(q_{z \|}-1.5 q_{z \perp}\right)+\frac{3}{5} \alpha_{z} J_{\|}\right] \\
& \mu_{z} \frac{\partial q_{z \perp}}{\partial t}=\cdots-\mu_{z} \nu_{z} \frac{5 / 2}{\kappa_{e}}\left[q_{z \perp}-1.28\left(q_{z \|}-1.5 q_{z \perp}\right)+\frac{2}{5} \alpha_{z} J_{\|}\right]
\end{aligned}
$$

with parallel current

$$
J_{\|}=\sum_{\mathrm{Sp}} a_{z} u_{z \|}
$$

and with numerical coefficients

$$
\alpha_{e}=0.71 \quad \kappa_{e}=3.2 \quad \pi_{e}=0.73 \quad \eta=0.51
$$

for electrons and

$$
\alpha_{i}=0 . \quad \kappa_{i}=3.9 \quad \pi_{i}=0.96
$$

for singly charged ions. Note the appearance of $\eta_{\|}$in the $u_{z \|}$-equation for all species, since the resistivity essentially adds to $\partial A_{G} / \partial t$, and that the thermoelectric coupling between $J_{\|}$ and the heat fluxes affects only the electrons. For other charge states the corresponding coefficients for ions may be found in Ref. [10]. 
Finally, the Landau damping model is added,

$$
\begin{aligned}
& \mu_{z} \frac{\partial q_{z \|}}{\partial t}=\cdots-\mu_{z} \sqrt{\tau_{z} / \mu_{z}}\left(1-0.125 q^{2} R^{2} \nabla_{\|}^{2}\right) q_{z \|} \\
& \mu_{z} \frac{\partial q_{z \perp}}{\partial t}=\cdots-\mu_{z} \sqrt{\tau_{z} / \mu_{z}}\left(1-0.125 q^{2} R^{2} \nabla_{\|}^{2}\right) q_{z \perp}
\end{aligned}
$$

which gives a finite-difference compatible version of the original by Hammett and Perkins [3] and their successors [1, 2], as explained in Ref. [15]. This completes the description of the six-moment gyrofluid model ("GEM" from Refs. [4, 14, 15]), now extended to incorporate finite gyroradius effects in the nonlinear magnetic fluctuation dynamics.

\section{A. Gyrofluid equations for collisionless reconnection}

Simplified two dimensional models are often used in studies of collisionless reconnection [27 $30,52-55]$. In terms of the geometry only the dynamics perpendicular to a prescribed

guide field is retained; in the language of this work this means only the nonlinear brackets are kept, with the linear parallel derivative incorporated into the magnetic nonlinearities, assuming a Cartesian coordinate system which is not aligned to the component of the magnetic field described by the shear. The standard "four field model" is an isothermal two fluid version of the equations keeping parallel velocities and densities. In fluid language it is [30]

$$
\begin{aligned}
\frac{d \Omega}{d t} & =\nabla_{\|} J_{\|} \\
\frac{d n_{e}}{d t} & =\nabla_{\|}\left(J_{\|}-u_{\|}\right) \\
\mu_{i} \frac{d u_{\|}}{d t} & =-\nabla_{\|} n_{e} \\
\beta_{e} \frac{\partial A_{\|}}{\partial t}+\mu_{e} \frac{d J_{\|}}{d t} & =\nabla_{\|}\left(n_{e}-\phi\right)
\end{aligned}
$$

where the vorticity and current are given by

$$
\Omega=\nabla_{\perp}^{2} \phi \quad J_{\|}=-\nabla_{\perp}^{2} A_{\|}
$$

and the nonlinear derivatives are given by

$$
\frac{d}{d t}=\frac{\partial}{\partial t}+[\phi,] \quad \nabla_{\|}=-\beta_{e}[\psi,]
$$


This is obviously related to the above gyrofluid model, with cold ions and with isothermal electrons, and no FLR effects. The simplified "two-field model" is then prescribed by neglecting $u_{\|}$and setting $n_{e}=\Omega$. The four field model is equivalent to the two-moment simplification of the gyrofluid model, with $J_{\|}=u_{\|}-v_{\|}$and $\Omega=n_{e}-n_{i}$, so that

$$
\begin{aligned}
\frac{d n_{e}}{d t} & =-\nabla_{\|} v_{\|} \\
\frac{d n_{i}}{d t} & =-\nabla_{\|} u_{\|} \\
\beta_{e} \frac{\partial A_{\|}}{\partial t}+\mu_{i} \frac{d u_{\|}}{d t} & =-\nabla_{\|} \phi \\
\beta_{e} \frac{\partial A_{\|}}{\partial t}-\mu_{e} \frac{d v_{\|}}{d t} & =-\nabla_{\|}\left(\phi-n_{e}\right)
\end{aligned}
$$

up to some $\mu_{e} / \mu_{i}$ corrections. Putting the FLR effects back in (with constant background temperatures) we then have the two moment equations for each species,

$$
\begin{aligned}
\frac{d n_{z}}{d t} & =-\nabla_{\|} u_{z \|} \\
\beta_{e} \frac{\partial A_{G}}{\partial t}+\mu_{z} \frac{d u_{z \|}}{d t} & =-\nabla_{\|}\left(\phi_{G}+\tau_{z} n_{z}\right)
\end{aligned}
$$

with nonlinear derivatives,

$$
\frac{d}{d t}=\frac{\partial}{\partial t}+\left[\phi_{G},\right] \quad \nabla_{\|}=-\beta_{e}\left[A_{G},\right]
$$

and FLR potentials,

$$
\phi_{G}=\Gamma_{1} \phi \quad A_{G}=\Gamma_{1} A_{\|}
$$

and with polarisation,

$$
\sum_{\mathrm{sp}} a_{z}\left[\Gamma_{1} n_{z}+\frac{\Gamma_{0}-1}{\tau_{z}} \phi\right]=0
$$

and induction (cf. Eqs. 134]135),

$$
\left(\sum_{z} a_{z}\left[\frac{\beta_{e}}{\mu_{z}} \Gamma_{1}^{2}\right]-\nabla_{\perp}^{2}\right) A_{\|}=\sum_{z} \frac{a_{z}}{\mu_{z}}\left[\Gamma_{1}\left(\beta_{e} A_{G}+\mu_{z} u_{z \|}\right)\right]
$$

Hence, we find that the four field model has this obvious generalised FLR version, through the two gyrofluid moments, and the two-field model is basically the same thing with the ion gyrofluid moment variables neglected on the basis that self consistent gradient drive is 
absent and $\beta_{e} / \mu_{i} \ll 1$. The foregoing correspondence was shown in Refs. [14, 15], in the context of tokamak microturbulence.

The obvious next step for reconnection with finite electron gyroradius modifications to the nonlinear magnetic field dynamics is to restore all six gyrofluid moment variables,

$$
\begin{gathered}
\frac{\partial n_{z}}{\partial t}+\left[\phi_{G}, n_{z}\right]+\left[\Omega_{G}, T_{z \perp}\right]=\beta_{e}\left[A_{G}, u_{z \|}\right]+\beta_{e}\left[\chi_{G}, q_{z \perp}\right] \\
\beta_{e} \frac{\partial A_{G}}{\partial t}+\mu_{z} \frac{\partial u_{z \|}}{\partial t}+\mu_{z}\left[\phi_{G}, u_{z \|}\right]+\mu_{z}\left[\Omega_{G}, q_{z \perp}\right] \\
=\beta_{e}\left[A_{G},\left(\phi_{G}+\tau_{z} p_{z \|}\right)\right]+\beta_{e}\left[\chi_{G},\left(\Omega_{G}+\tau_{z} T_{z \perp}\right)\right] \\
\frac{\partial T_{z \perp}}{\partial t}+\left[\phi_{G}, T_{z \perp}\right]+\left[\Omega_{G},\left(n_{z}+2 T_{z \perp}\right)\right]=\beta_{e}\left[A_{G}, q_{z \perp}\right]+\beta_{e}\left[\chi_{G},\left(u_{z \|}+2 q_{z \perp}\right)\right] \\
\mu_{z} \frac{\partial q_{z \|}}{\partial t}+\mu_{z}\left[\phi_{G}, q_{z \|}\right]=\frac{3}{2} \tau_{z}\left[A_{G}, T_{z \|}\right] \\
\beta_{e} \frac{\partial \chi_{G}}{\partial t}+\mu_{z} \frac{\partial q_{z \perp}}{\partial t}+\mu_{z}\left[\phi_{G}, q_{z \perp}\right]+\mu_{z}\left[\Omega_{G},\left(u_{z \|}+2 q_{z \perp}\right)\right]+\beta_{e}\left[\chi_{G}, q_{z \perp}\right] \\
=\tau_{z} \beta_{e}\left[A_{G}, T_{z \perp}\right]+\beta_{e}\left[\chi_{G},\left(\phi_{G}+\tau_{z} p_{z \|}\right)\right]+\beta_{e}\left[\chi_{G}, 2\left(\Omega_{G}+\tau_{z} T_{z \perp}\right)\right]
\end{gathered}
$$

with FLR potentials and polarisation and induction equations as in the full six-moment gyrofluid model (Eqs. 131-134) above.

\section{B. Constraints on magnetic geometry}

At this level the derivation of a set of equations actually used in a computation depends on assumptions made about the geometry. For 2D reconnection studies in a model containing a guide field the typical case is that a background homogeneous magnetic field $\mathbf{B}_{0}$ is prescribed, the coordinates of the computational domain describe the plane perpendicular to $\mathbf{B}_{0}$, and the components of the magnetic field in this plane, labelled $x y$, are determined by the evolving potential $A_{\|}$. For 3D magnetised plasma turbulence studies the coordinates are usually aligned to a background magnetic field with both curvature and shear. The derivation of gyrofluid equations does not depend on the use of either, or other, sort of model. However, the most important point is this: energetic consistency must be maintained. In this context the only requirement is that the tensor $\mathbf{F}_{0}$ defining the nonlinear brackets is antisymmetric 
and divergence free, and the vector $\mathcal{K}^{i}$ defining the curvature operator is divergence free. Although the equations were derived using strict fluxtube ordering, that was made necessary by the dependence of $F^{M}$ upon $B$ and therefore its spatial dependence. That was simply an energetic consistency constraint. Once the equations are derived, one need only maintain that same level of energetic consistency, and this is done by retaining the required properties for $\mathbf{F}_{0}$ and $\mathcal{K}$. This makes a global geometry model possible. Local and global field aligned geometry is given in Refs. [35, 56] and the necessary constraints of global consistency of fluxtubes and the transforms necessary to obtain global mode structure are given in Refs. [36] and [37], respectively. An example of a global tokamak geometry model which relaxes the ordering on the coordinate derivatives, while keeping it on the equations themselves, is to recast the curvature operator in terms of a bracket,

$$
\mathcal{K}(f)=2[\log R, f]
$$

with $\log R$ a scalar function of the coordinates, and the brackets given as in Eq. 129 with $\log R$ and the components of $\mathbf{F}_{0}$, along with the metric and magnetic field component $B^{s}$, describing the geometry. A version of this is given in Ref. [37] and is suitable for global computation of the tokamak core, or the edge region within one scale-length variation of the background parameters dependent upon densities and temperatures. The ordering behind the equations essentially limits validity to a domain comprising one such set of scale lengths. Otherwise, the parameter set ceases to be representative (cf. the discussion in Refs. [14], [57], or [58]). These steps are what extend the GEM model of Refs. [4, 14, 15] to the GEMR model described in Refs. [51, 59].

\section{Global computation and the issue of stratification}

Global computations with this delta-f gyrofluid model and its predecessor in Ref. [15] are not only possible but have been underway for some time, both for edge [51, 60] and for core [59] cases. With the background current profile added to the electron parallel velocity dependent variable $u_{e \|} \rightarrow u_{e \|}-J_{0} /\left(n_{e} e\right)$, where $J_{0}=J_{0}(x)$, with $n_{e} e$ a normalising parameter, the model is being used for studies of the self consistent interaction between dominantly ion temperature gradient driven electromagnetic core turbulence with magnetic islands [61]. One often thinks of fluxtube computations as being defined by periodic radial 
boundary conditions. In fact they are defined by taking the radially local approximation on the flux surface geometry, so that all metric quantities depend on $s$ only except for the appearance of shear, and then assuming that $\partial / \partial x, \partial / \partial y \gg \partial / \partial s$ in the derivatives. (cf. Sec. IIB). Fluxtube computations without periodic boundary conditions are common (e.g., Refs. [4, 14, 47, 58, 62 64]). Although the delta-f gyrofluid equations are initially derived using fluxtube approximations on the gyrokinetic model, in the resulting gyrofluid model global geometry may be restored as long as the model drift tensor used to define the brackets is divergence free (cf. the previous subsection; this step cannot be taken directly on the delta-f gyrokinetic model, due to the dependence of $F^{M}$ upon $B$ and the need to commute $F^{M}$ past derivatives to conserve free energy).

The other issue faced by truly global computations is stratification. Although delta-f models can use global geometry and do global simulations, what they cannot represent is a change in physical parameters across the domain. Again, this is for energy conservation reasons: the physical parameters in the delta-f equations (including those in $F^{M}$ ) must also commute with the nonlinear bracket operations, including the $\mathcal{K}$ terms. A stratified nonlinear model will require that all locally varying parameters (i.e., temperature, beta) be determined (three-dimensionally) by the dependent variables. Then, the conserved quantity is no longer free energy but the Noether energy, as explained in Sec. IIC. For a gyrofluid model to be able to represent stratification, analogous to nonlocal fluid Braginskii equations of the sort given in Ref. [65], it must either be derived from a total-f gyrokinetic model such as given in Sec. II , or the simplified version without FLR effects used to treat equilibrium flows in Ref. [66], or directly from a model Lagrangian as in Refs. [18, 19]. This is still ongoing work as the need to have parallel heat fluxes as dynamical variables and the simultaneous incorporation of FLR effects requires extension of Refs. [18, 19], which is the closest at present towards a complete total-f gyrofluid model.

With such a stratified model one can anticipate to capture the spatial transition between regions of different physical character and perhaps also temporal transitions. Of course, to treat such phenomena one requires a solid foundation within energetic consistency. 


\section{Relation to dissipative fluid drift equations and Reduced MHD}

A detailed analysis of these dissipation terms under the Braginskii limits (small gyroradius, strong collisionality, and an implicit assumption that specific heat fluxes are small compared to fluid velocities) showed that they recover the forms used in reduced (low frequency, neglecting compressional Alfvén dynamics and expressing perpendicular flows as drifts rather than directly as dependent variables) Braginskii equations. Polarisation heat flux effects due to finite perpendicular inertia were also recovered. By extension, the reduced magnetohydrodynamic (MHD) equations are already well known to be a subset of the reduced Braginskii equations. These results were obtained in a previous work [67], and are summarised here.

The Braginskii regime is defined by the assumptions used by Braginskii to obtain his collisional fluid equations [10], whose low frequency regime still represents the most commonly used model for nonlinear edge turbulence computations. The assumptions are: (1) dominance by collisions, such that both $\nu_{z} \gg \partial / \partial t$ and $\nu_{z} \gg V_{z} \nabla_{\|}$, where $\nu_{z}$ is the like particle

collision frequency and $V_{z}$ is the thermal velocity, (2) long wavelength, such that $\rho_{z}^{2} \nabla_{\perp}^{2} \ll 1$, where $\rho_{z}$ is the thermal gyroradius, and, much less commonly understood, (3) small specific heat flux, such that $\mathbf{q}_{z} \ll p_{z} \mathbf{u}_{z}$, implicitly assumed in the use of a drifting Maxwellian to lowest order and obtaining $\mathbf{q}$ only through first order corrections. In these inequalities the subscript $z$ denotes the species, i.e., the inequalities should hold for all species. The most important thing to know about tokamak edge turbulence (in particular) is that all three of these assumptions are violated, and the problems are especially severe in the ions, not the electrons which received most of the early attention [50]. Specifically, $\nu_{i}$ is one to two orders of magnitude slower than the turbulence, for the longer and shorter wavelength component, respectively, and the turbulence vorticity spectrum always extends past $k_{\perp} \rho_{s}=1$ for drift wave turbulence (in a plasma with $T_{i} \sim T_{e}$ this means a component with $k_{\perp} \rho_{i} \sim 1$ must be faced), and especially for temperature gradient driven turbulence, not only are specific parallel heat fluxes larger than velocities in fluctuations, but the diamagnetic compressional effects in tokamak geometry scale with the gradients and therefore the heat flux effects are stronger in both the diamagnetic and polarisation effects in the ions. The latter indicates extending the fluid models to treat heat fluxes as well as velocities in the stress tensor effects that represent nonlinear polarisation, as introduced and discussed in Ref. [68]. 
The gyrofluid equations represent all these extension effects as well as the basic Braginskii ones automatically. Briefly, nonlinear finite gyroradius effects in the gyrofluid model reduce term by term to the nonlinear polarisation velocity and heat flux effects in an expansion keeping lowest order $k_{\perp}^{2} \rho_{z}^{2}$ effects in the ExB advection and in the equivalence of representation implied by the polarisation equation. For an isothermal model, one can start with the definition of $\phi_{G}$, solve the polarisation equation for $n_{i}$ in terms of $n_{e}$, expand these expressions one order in $k_{\perp}^{2} \rho_{z}^{2}$ and insert them into the nonlinear advection derivative for $n_{i}$, and do some operations involving converting $\nabla_{\perp}^{2}$ inside brackets into general divergences. The result is the fluid density equation with the nonlinear polarisation terms. With the temperatures the procedure is more complicated and involves manipulation of the $\Omega_{G}$ terms and derivation of the space/gyrofluid temperature representation from the moment hierarchy. This procedure recovers the temperature gradient parts of the polarisation velocity and also the polarisation heat flux terms.

The other correspondences involve collisional dissipation formulae and are simpler. The parallel viscosity is proportional to the temperature anisotropy $\delta T_{z}=T_{z \|}-T_{z \perp}$. The two temperature equations are used to form a time dependent equation for $\delta T_{z}$. The velocity divergences $\left(\nabla_{\|}\right.$and $\mathcal{K}$ terms $)$ and collisional dissipation are put on the right side, and the time derivatives, nonlinearities, and heat flux divergences are put on the left side. The Braginskii regime consists of assuming the left side to be small, recovering the collisional formula for parallel viscosity (since we set the $\pi_{z}$ coefficients accordingly). As noted, this is severely violated for ions in edge turbulence. This result was also obtained in Ref. [51].

The parallel heat flux in a collisional model is the sum of the perp-parallel and parallelparallel components. The equation for these are added, the temperature gradients and collisional damping terms are put on the right side and all other terms are put on the left side. The Braginskii regime consists of assuming the left side to be small, recovering the collisional formula for parallel heat flux (for this we set the $\kappa_{z}$ coefficients accordingly). This is also violated for ions, with the ExB advection nonlinearities much larger than any of the dissipation terms for typical parameters. This result was also obtained in Ref. [51].

Once the Braginskii regime is (formally) recovered, the next steps to reduced MHD are well known (this is a matter of the difference between two-fluid models, e.g., WakataniHasegawa [23, 69], and the one-fluid versions of reduced MHD [70, 71]). At the isothermal level, leading to what are called four-field models, this was done in detail with both models 
and results for edge turbulence in Ref. [72]. The basic assumptions are that $e \widetilde{\phi} / T_{e}$ is larger than any relative fluctuation variable in densities or temperatures, and that compressibility effects in $J_{\|}$are neglected (energetically, the second assumption follows from the first). This can be called MHD ordering (pressure gradients are neglected in favour of electric field components).

At the philosophical level, the gyrofluid model treats polarisation densities instead of velocities, but the simplest road back to reduced MHD is to apply $\partial / \partial t$ to the polarisation equation, apply MHD ordering, and follow all the consequences. Similarly, the Ohm's law amounts to neglecting electron inertia effects and applying MHD ordering to the electron parallel velocity equation. Reduced MHD without polarisation effects is particularly easy to understand (neglect all FLR effects, subtract the continuity equations, and replace the gyrocenter charge density with the ExB vorticity using the polarisation effects). A more introductory version of this correspondence between models was given in Ref. [14]. Basically, the gyrofluid model solves the same problems as the reduced Braginskii fluid model, without the more damaging assumptions of the latter.

\section{SUMMARY AND COMMENT}

The gyrofluid model corresponding to what is known as the "delta-f" gyrokinetic theory now has a derivation path which starts from first principles. It depends on the ordering used to obtain the delta-f forms of both the gyrokinetic equation and the associated field potential equations. It further depends on a list of moment variables, the description of the underlying distribution function in terms of these variables, and essentially one assumption involving the moment closure of finite gyroradius (FLR) corrections. The energy theorem descends from both the total- and delta-f gyrokinetic versions and is used to fix the rest of the undetermined quantities in the gyrofluid model. The resulting gyrofluid model is now consistent at the level of the best delta-f gyrokinetic models, especially in terms of an energy theorem which is properly conservative in all the reactive effects (compressibility, coupling to flows and MHD, etc.), and also in terms of the responses of heat fluxes to temperature gradients being carried at the same level of sophistication as the responses of flows and currents to density gradients and electric fields. For dynamics of magnetised plasmas driven principally by temperature gradients this is the minimal fluid model. For 
dynamics more generically pressure or current driven the isothermal version [14], easily and consistently obtained by setting the temperature and heat flux moment variables, the second FLR operation $\Gamma_{2}$, and the thermoelectric collisional effect (through $\alpha_{e}$ ) to zero, becomes the minimal model. Hence, the decision of what constitutes the minimal model depends on the problem being considered. However, the procedure given herein is useful generally in constraining the derivation of any gyrofluid model based upon, as it must be, an underlying gyrokinetic model which is energetically consistent.

[1] M. A. Beer and G. Hammett, Phys. Plasmas 3, 4046 (1996).

[2] W. Dorland and G. Hammett, Phys. Fluids B 5, 812 (1993).

[3] G. W. Hammett and F. W. Perkins, Phys. Rev. Lett. 64, 3019 (1990).

[4] B. Scott, Phys. Plasmas 7, 1845 (2000).

[5] P. Snyder and G. Hammett, Phys. Plasmas 8, 744 (2001).

[6] G. Knorr, F. R. Hansen, J. P. Lynov, H. L. Pécseli, and J. J. Rasmussen, Physica Scripta 38, 829 (1988).

[7] W. W. Lee, Phys. Fluids 26, 556 (1983).

[8] E. A. Frieman and L. Chen, Phys. Fluids 25, 502 (1982).

[9] H. Grad, Commun. Pure Appl. Math. 2, 331 (1949).

[10] S. I. Braginskii, Rev. Plasma Phys. 1, 205 (1965).

[11] R. Littlejohn, J. Plasma Phys. 29, 111 (1983).

$[12]$ D. H. E. Dubin, J. A. Krommes, C. Oberman, and W. W. Lee, Phys. Fluids 26, 3524 (1983).

[13] T. S. Hahm, Phys. Fluids 31, 2670 (1988).

[14] B. Scott, Plasma Phys. Contr. Fusion 45, A385 (2003).

[15] B. Scott, Phys. Plasmas 12, 102307 (2005), arXiv:physics/0501124.

[16] H. Sugama, Phys. Plasmas 7, 466 (2000).

[17] A. Brizard, Phys. Plasmas 7, 4816 (2000).

[18] D. Strintzi and B. Scott, Phys. Plasmas 11, 5452 (2004).

[19] D. Strintzi, B. Scott, and A. Brizard, Phys. Plasmas 12, 052517 (2005).

[20] W. W. Lee and W. M. Tang, Phys. Fluids 31, 612 (1988).

[21] A. Hasegawa and K. Mima, Phys. Fluids 21, 87 (1978). 
[22] D. Montgomery and L. Turner, Phys. Fluids 23, 264 (1980).

[23] M. Wakatani and A. Hasegawa, Phys. Fluids 27, 611 (1984).

[24] B. Scott, Phys. Fluids B 4, 2468 (1992).

[25] J. A. Krommes and G. Hu, Phys. Plasmas 1, 3211 (1994).

[26] H. Sugama, T. Watanabe, and W. Horton, Phys. Plasmas 8, 2617 (2001).

[27] F. Porcelli, Phys. Rev. Lett. 66, 425 (1991).

[28] T. J. Schep, F. Pegoraro, and B. N. Kuvshinov, Phys. Plasmas 1, 2843 (1994).

[29] D. Grasso, F. Pegoraro, F. Porcelli, and F. Califano, Plasma Phys. Contr. Fusion 41, 1497 (1999).

[30] B. Scott and F. Porcelli, Phys. Plasmas 11, 5468 (2004).

[31] V. Grandgirard, Y. Sarazin, P. Angelino, A. Bottino, N. Crouseilles, G. Darmet, G. DifPradalier, X. Garbet, P. Ghendrih, S. Jolliet, et al., Plasma Phys. Contr. Fusion 49, B173 (2007).

[32] Y. Idomura, M. Ida, S. Tokuda, and L. Villard, J. Comput. Phys. 226, 244 (2007).

[33] X. Garbet, Y. Sarazin, V. Grandgirard, G. Dif-Pradalier, G. Darmet, P. Ghendrih, P. Angelino, P. Bertrand, N. Besse, E. Gravier, et al., Nucl. Fusion 47, 1206 (2007).

[34] T. S. Hahm, W. W. Lee, and A. Brizard, Phys. Fluids 31, 1940 (1988).

[35] M. Beer, S. Cowley, and G. Hammett, Phys. Plasmas 2, 2687 (1995).

[36] B. Scott, Phys. Plasmas 5, 2334 (1998).

[37] B. Scott, Phys. Plasmas 8, 447 (2001).

[38] R. Gagne and M. M. Shoucri, J. Comput. Phys. 24, 445 (1977).

[39] M. M. Shoucri, Phys. Fluids 21, 1359 (1978).

[40] A. Ghizzo, B. Izrar, P. Bertrand, E. Fijalkow, M. R. Feix, and M. Shoucri, Phys. Fluids 31, 72 (1988).

[41] P. Bertrand, A. Ghizzo, T. W. Johnston, M. Shoucri, E. Fijalkow, and M. R. Feix, Phys. Fluids B 2, 1028 (1990).

[42] T. Dannert and F. Jenko, Comput. Phys. Comm. 163, 67 (2004).

[43] T. Dannert and F. Jenko, Phys. Plasmas 12, 07239 (2005).

[44] A. Brizard, Phys. Plasmas 11, 4429 (2004).

[45] A. Mishchenko and A. Könies, J. Plasma Phys. 73, 757 (2007).

[46] B. Scott, Plasma Phys. Contr. Fusion 48, A387 (2006). 
[47] B. Scott, Plasma Phys. Contr. Fusion 39, 1635 (1997).

[48] X. Q. Xu and R. H. Cohen, Contrib. Plasma Phys. 38, 158 (1998).

[49] B. N. Rogers, J. F. Drake, and A. Zeiler, Phys. Rev. Lett. 81, 4396 (1998).

[50] A. B. Hassam, Phys. Fluids 23, 38 (1980).

[51] B. Scott, Contrib. Plasma Phys. 46, 714 (2006).

[52] M. Ottaviani and F. Porcelli, Phys. Rev. Lett. 71, 3802 (1993).

[53] M. Ottaviani and F. Porcelli, Phys. Plasmas 2, 4104 (1995).

[54] R. Fitzpatrick and F. Porcelli, Phys. Plasmas 11, 4713 (2004).

[55] R. Fitzpatrick and F. Porcelli, Phys. Plasmas 14, 049902 (2007).

[56] R. L. Dewar and A. H. Glasser, Phys. Fluids 26, 3038 (1983).

[57] B. Scott, Plasma Phys. Contr. Fusion 48, B277 (2006).

[58] B. Scott, Plasma Phys. Contr. Fusion 49, S25 (2007).

[59] G. L. Falchetto, B. D. Scott, P. Angelino, A. Bottino, T. Dannert, V. Grandgirard, S. J. Janhunen, F. Jenko, S. Jolliet, A. Kendl, et al., Plasma Phys. Contr. Fusion 50, 124015 (2008).

[60] S. J. Zweben, B. D. Scott, J. L. Terry, B. LaBombard, J. W. Hughes, and D. P. Stotler, Phys. Plasmas 16, 082505 (2009).

[61] E. Poli, A. Bottino, W. A. Hornsby, A. G. Peeters, T. Ribeiro, B. D. Scott, and M. Siccino, Plasma Phys. Contr. Fusion 52, submitted (2010).

[62] V. Naulin, Phys. Plasmas 10, 4016 (2003).

[63] V. Naulin, A. Kendl, O. E. Garcia, A. H. Nielsen, and J. J. Rasmussen, Phys. Plasmas 12, $052515(2005)$.

[64] B. Scott, New J. Phys. 7, 92 (2005).

[65] B. Scott, Phys. Plasmas 10, 963 (2003).

[66] B. Scott, A. Kendl, and T. Ribeiro, Contrib. Plasma Phys. 50, 228 (2010).

[67] B. Scott, Phys. Plasmas 14, 102318 (2007).

[68] I. O. Pogutse, A. I. Smolyakov, and A. Hirose, J. Plasma Phys. 60, 133 (1998).

[69] A. Hasegawa and M. Wakatani, Phys. Rev. Lett. 50, 682 (1983).

[70] H. Strauss, Phys. Fluids 19, 134 (1976).

[71] H. Strauss, Phys. Fluids 20, 1354 (1977).

[72] B. Scott, Phys. Plasmas 12, 062314 (2005). 